\title{
Solvability of a Third-Order Singular Generalized Left Focal Problem in Banach Spaces
}

\author{
Youwei Zhang \\ Department of Mathematics, Hexi University, Gansu 734000, China \\ Correspondence should be addressed to Youwei Zhang; ywzhang0288@163.com
}

Received 9 February 2014; Accepted 5 May 2014; Published 26 May 2014

Academic Editor: Chuanxi Qian

Copyright (c) 2014 Youwei Zhang. This is an open access article distributed under the Creative Commons Attribution License, which permits unrestricted use, distribution, and reproduction in any medium, provided the original work is properly cited.

We consider the existence of positive solution for a third-order singular generalized left focal boundary value problem with full derivatives in Banach spaces. Green's function and its properties, explicit a priori, estimates will be presented. By means of the theories of the fixed point in cones, we establish some new and general results on the existence of single and multiple positive solutions to the third-order singular generalized left focal boundary value problem. Our results are generalizations and extensions of the results of the focal boundary value problem. An example is included to illustrate the results obtained.

\section{Introduction}

Third-order differential equation describes many phenomena in applied mathematics, physical science, aeronautics, and applied mechanics such as the study of steady flows produced by free jets, wall jets, liquid jets, the flow past a stretching plate, and Blasius flow [1-6]. For two-dimensional flow of a fluid with small viscosity adhering to the flat plate, the simplified version, which has been derived by Blasius, only uses two unknowns $u$ and $v$. Laminar flat plate flow across a flat plate can be expressed using a boundary-layer equation defined by Blasius. Simplified momentum equation is given by

$$
\begin{gathered}
u \frac{\partial u}{\partial x}+v \frac{\partial v}{\partial y}=v \frac{\partial^{2} u}{\partial y^{2}}, \\
u(x, 0)=v(x, 0)=0, \quad u(x, \infty)=U_{\infty},
\end{gathered}
$$

where $u=u(x, y)$ is the velocity measured, $v=v(x, y)$ is the transverse velocity component, $x$ is length of the plate, $y$ is the distance away from the plate, and $U_{\infty}$ is free-stream velocity.
Assume that the leading edge of the plate is $x=0$ and the plate is infinity long; this equation can be simplified as

$$
-\frac{U_{\infty}^{2}}{2 x} \varsigma f^{\prime} f^{\prime \prime}+\frac{U_{\infty}^{2}}{2 x}\left(\varsigma f^{\prime}-f\right)=v \frac{U_{\infty}^{2}}{x v} f^{\prime \prime \prime},
$$

where $f=f(\varsigma)$ is the dimensionless stream function. For further simplification, we have the Blasius differential equation

$$
f^{\prime} f^{\prime \prime}+2 f^{\prime \prime \prime}=0
$$

where the boundary conditions are $\varsigma=0, f=0$, and $f^{\prime}=0$ or $\varsigma=\infty, f^{\prime}=0$. For other related application results of the problem we refer to [7-10]. Recently, increasing attention is paid to the question of the solution for the third-order focal boundary value problem, especially for right focal problem. The main means are the Leray-Schauder continuation theorem, iteration of monotone mapping, upper and lower solutions method, fixed point theory, and so on. Many applications of the above tools of various nonlocal boundary conditions include recent works [11-13] and the reference therein. It is well known that a powerful tool for proving the existence of the solution to the focal boundary value problem is the fixed point theorem. In many cases, it is possible to find single or multiple solutions for the given problem. We 
would like to mention some results of $[14,15]$. Anderson [14] has investigated the existence of a solution to a third-order generalized right focal problem

$$
\begin{gathered}
x^{\prime \prime \prime}(t)=f(t, x(t)), \quad t_{1}<t<t_{3}, \\
x\left(t_{1}\right)=x^{\prime}\left(t_{2}\right)=0, \quad \gamma x\left(t_{3}\right)+\delta x^{\prime \prime}\left(t_{3}\right)=0, \\
t_{1}<t_{2}<t_{3},
\end{gathered}
$$

for certain constraints placed on nonnegative $f$; the main approach is the Leggett and Williams fixed point theorem. Minhós [15] has proved an Ambrosetti-Prodi type result for the third-order fully nonlinear right focal problem

$$
\begin{gathered}
u^{\prime \prime \prime}(t)+f\left(t, u(t), u^{\prime}(t), u^{\prime \prime}(t)\right)=s p(t), \\
0<t<1, \\
u(0)=A, \quad a u^{\prime}(0)-b u^{\prime \prime}(0)=B, \\
c u^{\prime}(1)+d u^{\prime \prime}(1)=C,
\end{gathered}
$$

where $f:[0,1] \times \mathbb{R} \rightarrow \mathbb{R}$ and $p:[0,1] \rightarrow \mathbb{R}^{+}$are continuous functions and $s \in \mathbb{R}$. The author has studied the existence, nonexistence, and multiplicity of solutions as variable $s$. Further, we may refer to recent contributions of Agarwal [16] and Agarwal et al. [17], Cabada and Heikkilä [18], and Wong and Agarwal [19] for the right focal problem. Accordingly, a third-order left focal problem for differential equation plays a role in many related fields too, even less attention has been focused, however, on third-order left focal problem. This paper will consider the existence of a positive solution for the following third-order singular generalized left focal boundary value problem with full derivatives in Banach spaces

$$
\begin{gathered}
u^{\prime \prime \prime}(t)+h(t) f\left(t, u(t), u^{\prime}(t), u^{\prime \prime}(t)\right)=0, \\
0<t_{1}<t<t_{2}, \\
a u\left(t_{1}\right)-b u^{\prime \prime}\left(t_{1}\right)=0, \\
u^{\prime}(\eta)=0, \quad t_{1}<\eta<t_{2}, \\
c u\left(t_{2}\right)+d u^{\prime}\left(t_{2}\right)=0,
\end{gathered}
$$

where $a, b, c, d, t_{1}, \eta, t_{2}, h$, and $f$ satisfy

$$
\begin{aligned}
& \left.\left(H_{1}\right) a, b, c, d \in\right] 0, \infty\left[\text { and } k=2 b c+2 a d\left(t_{2}-\eta\right)+a c\left(t_{2}-\right.\right. \\
& \left.t_{1}\right)\left(t_{1}+t_{2}-2 \eta\right)>0, \\
& \left(H_{2}\right) t_{1}<\eta<t_{2} \text { with } t_{2}-\eta>\eta-t_{1}, \\
& \left(H_{3}\right) h \in C(] t_{1}, t_{2}\left[,\left[0, \infty[) \text { may be singular at } t=t_{1}\right. \text { and/or }\right. \\
& \quad t=t_{2} \text { and } \int_{t_{1}}^{t_{2}} h(s) d s<\infty, \\
& \left(H_{4}\right) f \in C\left(\left[t_{1}, t_{2}\right] \times[0, \infty[\times]-\infty, \infty[2,[0, \infty[) .\right.
\end{aligned}
$$

The novelty of our problem is to show the existence of solution of the left focal boundary value problem; we will concentrate on the solvability of the third-order singular generalized left focal problem for differential equation. To the best of our knowledge, it is the first time that the thirdorder left focal problem has been discussed successfully. As one might expect, the technique of finding Green's function for the corresponding homogeneous left focal problem, as the kernel of an integral equation of Hammerstein type, has proven to be an effective method in most of thirdorder left focal problems. For this, we will employ known sign properties of Green's function and its partial derivative cleverly. Note that the solution $u$ of the problem $\left(\mathscr{P}_{s}\right)$ lacks concavity, which is slightly different from right focal problem. In order to overcome this difficulty, we will establish the appropriate a priori estimates of the solution. On the other hand, the condition on nonlinearity required in this paper is different from those of existing papers, which motivated us to consider the above left focal problem, since $f$ may be superlinear (sublinear). As usual, the function $f$ in the problem $\left(\mathscr{P}_{s}\right)$ is called superlinear if $\underline{f}_{0}=0$ and $\bar{f}_{\infty}=\infty$; it is called sublinear when $\underline{f}_{\infty}=0$ and $\overline{\bar{f}}_{0}^{0}=\infty$, where

$$
\begin{aligned}
& \underline{f}_{0}=\liminf _{\mu \rightarrow 0^{+}} \min _{t \in\left[t_{1}, t_{2}\right]} \frac{f(t, \mu, \nu, \omega)}{\mu}, \\
& \bar{f}_{\infty}=\limsup _{\mu \rightarrow \infty} \max _{t \in\left[t_{1}, t_{2}\right]} \frac{f(t, \mu, v, \omega)}{\mu}, \\
& \underline{f}_{\infty}=\liminf _{\mu \rightarrow \infty} \min _{t \in\left[t_{1}, t_{2}\right]} \frac{f(t, \mu, v, \omega)}{\mu}, \\
& \bar{f}_{0}=\limsup _{\mu \rightarrow 0^{+}} \max _{t \in\left[t_{1}, t_{2}\right]} \frac{f(t, \mu, \nu, \omega)}{\mu},
\end{aligned}
$$

for $\nu, \omega \in]-\infty, \infty[$. Based on the known results of the singular multipoint boundary value problem and high-order right focal problem for differential equation, this study is devoted to proving the existence of solution for the thirdorder singular generalized left focal problem $\left(\mathscr{P}_{s}\right)$, and the full derivatives are involved in the nonlinear term explicitly. Under the suitable limit and growth conditions on the nonlinearity $f$ in cones, we will establish some new and general results on the existence of single and multiple positive solutions to the problem $\left(\mathscr{P}_{s}\right)$; our results develop some results of the third-order focal problem and improve the results of third-order singular problem. The author believes that those results are useful in many applications for temporary new fields. Now we state the fixed point theorems.

Lemma 1 (see $[20,21])$. Let $K$ be a cone in a Banach space E. Assume that $\mathscr{K}\left(V_{1}\right), \mathscr{K}\left(V_{2}\right)$ are open subsets of $E$ with $0 \in$ $\mathscr{K}\left(V_{1}\right), \overline{\mathscr{K}\left(V_{1}\right)} \subset \mathscr{K}\left(V_{2}\right)$. If $\left.T: K \cap \overline{\left(\mathscr{K}\left(V_{2}\right)\right.} \backslash \mathscr{K}\left(V_{1}\right)\right) \rightarrow K$ is a completely continuous operator such that either

$\left(A_{1}\right)\|T x\| \leq\|x\|$ for all $x \in K \cap \partial \mathscr{K}\left(V_{1}\right)$ and $\|T x\| \geq\|x\|$ for all $x \in K \cap \partial \mathscr{K}\left(V_{2}\right)$ or

$\left(A_{2}\right)\|T x\| \geq\|x\|$ for all $x \in K \cap \partial \mathscr{K}\left(V_{1}\right)$ and $\|T x\| \leq\|x\|$ for all $x \in K \cap \partial \mathscr{K}\left(V_{2}\right)$.

Then, $T$ has a fixed point in $K \cap\left(\overline{\mathscr{K}\left(V_{2}\right)} \backslash \mathscr{K}\left(V_{1}\right)\right)$. 
Let $\gamma$ and $\theta$ be nonnegative continuous convex functionals on a cone $K$, let $\alpha$ be a nonnegative continuous concave functional on $K$, let $\psi$ be a nonnegative continuous functional on $K$, and let $m_{1}, m_{2}, m_{3}$, and $m_{4}$ be positive numbers. Define the convex sets as follows:

$$
\begin{gathered}
P\left(\gamma, m_{4}\right)=\left\{u \in K: \gamma(u)<m_{4}\right\}, \\
P\left(\gamma, \alpha, m_{2}, m_{4}\right)=\left\{u \in K: m_{2} \leq \alpha(u), \gamma(u) \leq m_{4}\right\}, \\
P\left(\gamma, \theta, \alpha, m_{2}, m_{3}, m_{4}\right) \\
=\left\{u \in K: m_{2} \leq \alpha(u), \theta(u) \leq m_{3}, \gamma(u) \leq m_{4}\right\},
\end{gathered}
$$

and a closed set

$$
Q\left(\gamma, \psi, m_{1}, m_{4}\right)=\left\{u \in K: m_{1} \leq \psi(u), \gamma(u) \leq m_{4}\right\} .
$$

Lemma 2 (see [22]). Let $K$ be a cone in a real Banach space $E$. Let $\gamma$ and $\theta$ be nonnegative continuous convex functionals on $K$, let $\alpha$ be a nonnegative continuous concave functional on $K$, and let $\psi$ be a nonnegative continuous functional on $K$ satisfying $\psi(\lambda u) \leq \lambda \psi(u)$ for $0 \leq \lambda \leq 1$ such that for some positive numbers $\epsilon$ and $m_{4}$

$$
\alpha(u) \leq \psi(u), \quad\|u\| \leq \epsilon \gamma(u),
$$

for all $u \in \overline{P\left(\gamma, m_{4}\right)}$. Suppose that $T: \overline{P\left(\gamma, m_{4}\right)} \rightarrow \overline{P\left(\gamma, m_{4}\right)}$ is completely continuous and there exist positive numbers $m_{1}, m_{2}$, and $m_{3}$ with $m_{1}<m_{2}$ such that

$\left(B_{1}\right)\left\{u \in P\left(\gamma, \theta, \alpha, m_{2}, m_{3}, m_{4}\right): \alpha(u)>m_{2}\right\} \neq \emptyset$ and $\alpha(T u)>m_{2}$ for $u \in P\left(\gamma, \theta, \alpha, m_{2}, m_{3}, m_{4}\right)$,

$\left(B_{2}\right) \alpha(T u)>m_{2}$ for $u \in P\left(\gamma, \alpha, m_{2}, m_{4}\right)$ with $\theta(T u)>m_{3}$,

$\left(B_{3}\right) 0 \notin Q\left(\gamma, \psi, m_{1}, m_{4}\right)$ and $\psi(T u)<m_{1}$ for $u \in$ $Q\left(\gamma, \psi, m_{1}, m_{4}\right)$ with $\psi(u)=m_{1}$.

Then, $T$ has at least three fixed points $u_{1}, u_{2}, u_{3} \in \overline{P\left(\gamma, m_{4}\right)}$ such that

$$
\begin{gathered}
\gamma\left(u_{i}\right) \leq m_{4} \quad \text { for } i=1,2,3, \\
m_{2}<\alpha\left(u_{1}\right),
\end{gathered}
$$

$m_{1}<\psi\left(u_{2}\right) \quad$ with $\alpha\left(u_{2}\right)<m_{2}, \quad \psi\left(u_{3}\right)<m_{1}$.

\section{Preliminaries}

We will employ several lemmas; these lemmas are based on the corresponding linear third-order generalized left focal boundary value problem.

Lemma 3. Let $\left(H_{1}\right),\left(H_{2}\right)$ hold. Green's function $G(t, s)$ for the boundary value problem

$$
\begin{gathered}
u^{\prime \prime \prime}(t)=0, \quad t_{1}<t<t_{2}, \\
a u\left(t_{1}\right)-b u^{\prime \prime}\left(t_{1}\right)=0, \\
u^{\prime}(\eta)=0, \quad t_{1}<\eta<t_{2}, \\
c u\left(t_{2}\right)+d u^{\prime}\left(t_{2}\right)=0
\end{gathered}
$$

is given as

$$
G(t, s)=\left\{\begin{array}{l}
s \in\left[t_{1}, \eta\right] \begin{cases}x_{1}(t, s), & s \leq t \\
y_{1}(t, s), & t \leq s,\end{cases} \\
s \in\left[\eta, t_{2}\right] \begin{cases}x_{2}(t, s), & s \leq t \\
y_{2}(t, s), & t \leq s,\end{cases}
\end{array}\right.
$$

where

$$
\begin{aligned}
x_{1} & (t, s) \\
= & \frac{1}{k}\left(b+\frac{a}{2}\left(t-t_{1}\right)^{2}-a\left(\eta-t_{1}\right)\left(t-t_{1}\right)\right) \\
& \times\left(c\left(t_{2}-s\right)^{2}+2 d\left(t_{2}-s\right)-2\left(c\left(t_{2}-t_{1}\right)+d\right)(\eta-s)\right) \\
& +\left(t-t_{1}\right)(\eta-s)-\frac{1}{2}(t-s)^{2}, \\
y_{1} & (t, s) \\
= & \frac{1}{k}\left(b+\frac{a}{2}\left(t-t_{1}\right)^{2}-a\left(\eta-t_{1}\right)\left(t-t_{1}\right)\right) \\
& \times\left(c\left(t_{2}-s\right)^{2}+2 d\left(t_{2}-s\right)-2\left(c\left(t_{2}-t_{1}\right)+d\right)(\eta-s)\right) \\
& +\left(t-t_{1}\right)(\eta-s), \\
x_{2} & (t, s) \\
= & \frac{1}{k}\left(b+\frac{a}{2}\left(t-t_{1}\right)^{2}-a\left(\eta-t_{1}\right)\left(t-t_{1}\right)\right) \\
& \times\left(c\left(t_{2}-s\right)^{2}+2 d\left(t_{2}-s\right)\right)-\frac{1}{2}(t-s)^{2}, \\
y_{2} & (t, s) \\
= & \frac{1}{k}\left(b+\frac{a}{2}\left(t-t_{1}\right)^{2}-a\left(\eta-t_{1}\right)\left(t-t_{1}\right)\right) \\
& \times\left(c\left(t_{2}-s\right)^{2}+2 d\left(t_{2}-s\right)\right) .
\end{aligned}
$$

Proof. First, we check that $G(t, s)$ is defined for each $(t, s) \in$ $\left[t_{1}, t_{2}\right] \times\left[t_{1}, t_{2}\right]$. Obviously, $x_{1}(s, s)=y_{1}(s, s), x_{2}(s, s)=$ $y_{2}(s, s)$. At $s=\eta$,

$$
\begin{aligned}
& x_{1}(t, \eta) \\
&= \frac{1}{k}\left(b+\frac{a}{2}\left(t-t_{1}\right)^{2}-a\left(\eta-t_{1}\right)\left(t-t_{1}\right)\right) \\
& \times\left(c\left(t_{2}-\eta\right)^{2}+2 d\left(t_{2}-\eta\right)\right)-\frac{1}{2}(t-\eta)^{2} \\
&= x_{2}(t, \eta), \\
& y_{1}(t, \eta) \\
&=\frac{1}{k}\left(b+\frac{a}{2}\left(t-t_{1}\right)^{2}-a\left(\eta-t_{1}\right)\left(t-t_{1}\right)\right) \\
& \quad \times\left(c\left(t_{2}-\eta\right)^{2}+2 d\left(t_{2}-\eta\right)\right) \\
&=y_{2}(t, \eta) .
\end{aligned}
$$


Next, we check that $G(t, s)$ satisfies the boundary conditions of problem (11). For convenience, we note that

$$
\begin{aligned}
& \frac{\partial}{\partial t} G(t, s) \\
& s \in\left[t_{1}, \eta\right]\left\{\begin{array}{c}
\frac{a}{k}(t-\eta)\left(\begin{array}{c}
c\left(t_{2}-s\right)^{2}+2 d\left(t_{2}-s\right) \\
\left.-2\left(c\left(t_{2}-t_{1}\right)+d\right)(\eta-s)\right) \\
+(\eta-t), \quad s \leq t,
\end{array}\right. \\
\frac{a}{k}(t-\eta)\left(c\left(t_{2}-s\right)^{2}+2 d\left(t_{2}-s\right)\right. \\
s \in\left[\eta, t_{2}\right]\left\{\begin{array}{cc}
\left.\left.-2\left(t_{2}-t_{1}\right)+d\right)(\eta-s)\right) \\
+(\eta-s), & t \leq s, \\
x\left(c\left(t_{2}-s\right)^{2}+2 d\left(t_{2}-s\right)\right)-(t-s), & s \leq t, \\
\frac{a}{k}(t-\eta)\left(c\left(t_{2}-s\right)^{2}+2 d\left(t_{2}-s\right)\right), & t \leq s,
\end{array}\right.
\end{array}\right.
\end{aligned}
$$

$$
\begin{aligned}
& \frac{\partial^{2}}{\partial t^{2}} G(t, s)
\end{aligned}
$$

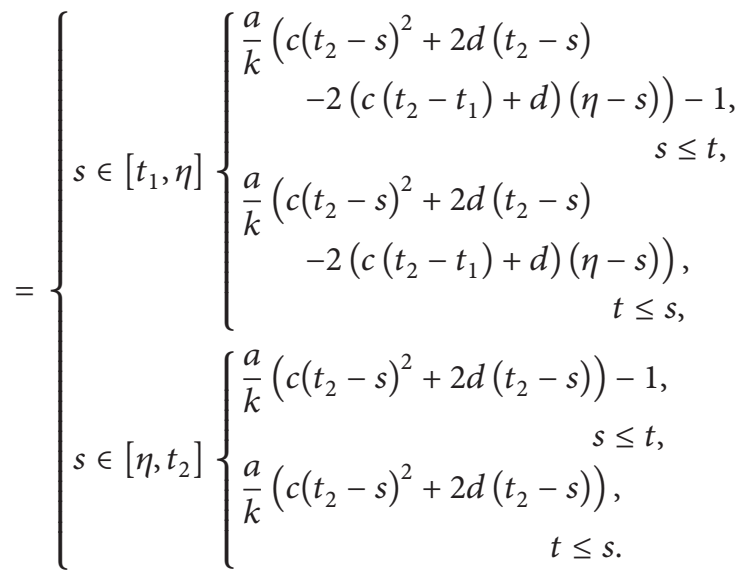

For $s \in\left[t_{1}, \eta\right]$, by (12) and (16), we have

$$
\begin{aligned}
& a G\left(t_{1}, s\right)-b \frac{\partial^{2}}{\partial t^{2}} G\left(t_{1}, s\right) \\
& =a y_{1}\left(t_{1}, s\right)-b \frac{\partial^{2}}{\partial t^{2}} y_{1}\left(t_{1}, s\right) \\
& =\frac{a b}{k}\left(c\left(t_{2}-s\right)^{2}+2 d\left(t_{2}-s\right)-2\left(c\left(t_{2}-t_{1}\right)+d\right)(\eta-s)\right) \\
& \quad-\frac{a b}{k}\left(c\left(t_{2}-s\right)^{2}+2 d\left(t_{2}-s\right)-2\left(c\left(t_{2}-t_{1}\right)+d\right)(\eta-s)\right) \\
& =0 ;
\end{aligned}
$$

for $s \in\left[\eta, t_{2}\right]$, we get

$$
\begin{aligned}
& a G\left(t_{1}, s\right)-b \frac{\partial^{2}}{\partial t^{2}} G\left(t_{1}, s\right) \\
& =a y_{2}\left(t_{1}, s\right)-b \frac{\partial^{2}}{\partial t^{2}} y_{2}\left(t_{1}, s\right) \\
& =\frac{a b}{k}\left(c\left(t_{2}-s\right)^{2}+2 d\left(t_{2}-s\right)\right) \\
& \quad-\frac{a b}{k}\left(c\left(t_{2}-s\right)^{2}+2 d\left(t_{2}-s\right)\right) \\
& =0 .
\end{aligned}
$$

For $s \in\left[t_{1}, \eta\right]$, by (12) and (15), we have $(\partial / \partial t) G(\eta, s)=$ $(\partial / \partial t) x_{1}(\eta, s)=0$; for $s \in\left[\eta, t_{2}\right]$, we get $(\partial / \partial t) G(\eta, s)=$ $(\partial / \partial t) y_{2}(\eta, s)=0$

For $s \in\left[t_{1}, \eta\right]$, by (12) and (15), we have

$$
\begin{aligned}
c G & \left(t_{2}, s\right)+d \frac{\partial}{\partial t} G\left(t_{2}, s\right) \\
= & c x_{1}\left(t_{2}, s\right)+d \frac{\partial}{\partial t} x_{1}\left(t_{2}, s\right) \\
= & \frac{c}{k}\left(b+\frac{a}{2}\left(t_{2}-t_{1}\right)^{2}-a\left(\eta-t_{1}\right)\left(t_{2}-t_{1}\right)\right) \\
& \times\left(c\left(t_{2}-s\right)^{2}+2 d\left(t_{2}-s\right)-2\left(c\left(t_{2}-t_{1}\right)+d\right)(\eta-s)\right) \\
& +c\left(t_{2}-t_{1}\right)(\eta-s)-\frac{c}{2}\left(t_{2}-s\right)^{2} \\
& +\frac{a d}{k}\left(t_{2}-\eta\right) \\
& \times\left(c\left(t_{2}-s\right)^{2}+2 d\left(t_{2}-s\right)-2\left(c\left(t_{2}-t_{1}\right)+d\right)(\eta-s)\right) \\
& +d\left(\eta-t_{2}\right) \\
= & \frac{c}{2}\left(t_{2}-s\right)^{2}+d\left(t_{2}-s\right) \\
& -\left(c\left(t_{2}-t_{1}\right)+d\right)(\eta-s)+c\left(t_{2}-t_{1}\right)(\eta-s) \\
& -\frac{c}{2}\left(t_{2}-s\right)^{2}+d\left(\eta-t_{2}\right) \\
& 0 ;
\end{aligned}
$$

for $s \in\left[\eta, t_{2}\right]$, we get

$$
\begin{aligned}
& c G\left(t_{2}, s\right)+d \frac{\partial}{\partial t} G\left(t_{2}, s\right) \\
& =c x_{2}\left(t_{2}, s\right)+d \frac{\partial}{\partial t} x_{2}\left(t_{2}, s\right)
\end{aligned}
$$




$$
\begin{aligned}
= & \frac{c}{k}\left(b+\frac{a}{2}\left(t_{2}-t_{1}\right)^{2}-a\left(\eta-t_{1}\right)\left(t_{2}-t_{1}\right)\right) \\
& \times\left(c\left(t_{2}-s\right)^{2}+2 d\left(t_{2}-s\right)\right)-\frac{c}{2}\left(t_{2}-s\right)^{2} \\
& +\frac{a d}{k}\left(t_{2}-\eta\right) \\
& \times\left(c\left(t_{2}-s\right)^{2}+2 d\left(t_{2}-s\right)\right)+d\left(s-t_{2}\right) \\
= & \frac{c}{2}\left(t_{2}-s\right)^{2}+d\left(t_{2}-s\right)-\frac{c}{2}\left(t_{2}-s\right)^{2}+d\left(s-t_{2}\right) \\
= & 0 .
\end{aligned}
$$

Hence, $G(t, s)$ satisfies the boundary conditions of problem (11).

Lemma 4. Let $\left(H_{1}\right),\left(H_{2}\right)$ hold. If $b>(a / 2)\left(t_{2}-t_{1}\right)^{2}$, then Green's function as given in (12) satisfies

$$
\begin{gathered}
G(t, s) \geq 0, \\
G(t, s) \geq \tau_{1} G(\eta, s), \\
\left|\frac{\partial}{\partial t} G(t, s)\right| \geq \tau_{2} \frac{\partial}{\partial t} G(\eta, s), \\
(t, s) \in\left[t_{1}, t_{2}\right]^{2},
\end{gathered}
$$

where

$$
\begin{aligned}
& \left.\tau_{1}=\min \left\{\tau_{11}, \tau_{12}\right\} \in\right] 0,1[, \\
& \tau_{11}=\min \left\{\min _{s \in\left[t_{1}, \eta\right]} \frac{x_{1}\left(t_{1}, t_{1}\right)}{x_{1}(\eta, s)}, \min _{s \in\left[\eta, t_{2}\right]} \frac{x_{2}\left(t_{2}, s\right)}{x_{2}(\eta, s)}\right\}, \\
& \tau_{12}=\min \left\{\min _{s \in\left[t_{1}, \eta\right]} \frac{y_{1}(\eta, \eta)}{y_{1}\left(t_{1}, s\right)}, \min _{s \in\left[\eta, t_{2}\right]} \frac{y_{2}(\eta, s)}{y_{2}\left(t_{2}, s\right)}\right\}, \\
& \tau_{2}=\min \left\{\min _{s \in\left[t_{1}, \eta\right]}\left|\frac{(\partial / \partial t) G(s, s)}{(\partial / \partial t) G\left(t_{1}, s\right)}\right|,\right. \\
& \left.\left.\min _{s \in\left[\eta, t_{2}\right]}\left|\frac{(\partial / \partial t) G(s, s)}{(\partial / \partial t) G\left(t_{2}, s\right)}\right|\right\} \in\right] 0,1[.
\end{aligned}
$$

Proof. Condition $b>(a / 2)\left(t_{2}-t_{1}\right)^{2}$ implies that $b>$ $(a / 2)\left(\eta-t_{1}\right)^{2}$ and $b+(a / 2)\left(t_{2}-t_{1}\right)^{2}-a\left(\eta-t_{1}\right)\left(t_{2}-t_{1}\right)>0$ for $t_{1}<\eta<t_{2}$. To prove the nonnegative property of Green's function, combining with (15) and (16), we divide it into two cases.

Case 1. For $s \leq t$,

$$
\begin{aligned}
\frac{\partial}{\partial t} G(t, s)= & \frac{\partial}{\partial t} x_{1}(t, s) \\
= & \frac{a}{k}(t-\eta) \\
& \times\left(c\left(t_{2}-s\right)^{2}+2 d\left(t_{2}-s\right)\right. \\
& \left.\quad-2\left(c\left(t_{2}-t_{1}\right)+d\right)(\eta-s)\right)+(\eta-t)
\end{aligned}
$$

$$
\begin{aligned}
& =\frac{c(t-\eta)}{k}\left(a\left(s-t_{1}\right)^{2}-2 b\right) \\
& \geq 0 \\
\frac{\partial^{2}}{\partial t^{2}} G(t, s) & =\frac{\partial^{2}}{\partial t^{2}} x_{1}(t, s) \\
& =\frac{a}{k}\left(c\left(t_{2}-s\right)^{2}+2 d\left(t_{2}-s\right)\right. \\
& \left.\quad-2\left(c\left(t_{2}-t_{1}\right)+d\right)(\eta-s)\right)-1
\end{aligned}
$$$$
\leq 0
$$

imply that $x_{1}(t, s)$ is nondecreasing and concave in $t$ on $\left[t_{1}, \eta\right]$; it follows that $G(\eta, s) \geq G(t, s)$ for each $(t, s) \in\left[t_{1}, \eta\right] \times\left[t_{1}, \eta\right]$ :

$$
\begin{aligned}
& G\left(t_{1}, s\right)= x_{1}\left(t_{1}, s\right) \\
&= \frac{b}{k}\left(c\left(t_{2}-s\right)^{2}+2 d\left(t_{2}-s\right)\right. \\
&\left.\quad-2\left(c\left(t_{2}-t_{1}\right)+d\right)(\eta-s)\right)-\frac{1}{2}\left(t_{1}-s\right)^{2} \\
&= \frac{b}{k}\left(c\left(t_{2}-s\right)^{2}+2 d\left(t_{2}-s\right)\right. \\
& \geq\left.0, \quad-2\left(c\left(t_{2}-t_{1}\right)+d\right)(\eta-s)\right) \\
& \frac{\partial}{\partial t} G(t, s)>0, \quad \frac{\partial}{\partial t} G(s, s)>0, \quad \frac{\partial}{\partial t} G\left(t_{1}, s\right)>0
\end{aligned}
$$

Likewise,

$$
\begin{aligned}
\frac{\partial}{\partial t} G(t, s)= & \frac{\partial}{\partial t} x_{2}(t, s) \\
= & \frac{a}{k}(t-\eta)\left(c\left(t_{2}-s\right)^{2}+2 d\left(t_{2}-s\right)\right)-(t-s) \\
= & (t-\eta)\left(\frac{a}{k}\left(c\left(t_{2}-s\right)^{2}+2 d\left(t_{2}-s\right)\right)-1\right) \\
& +(s-\eta) \\
\leq & 0, \\
\frac{\partial^{2}}{\partial t^{2}} G(t, s)= & \frac{\partial^{2}}{\partial t^{2}} x_{2}(t, s) \\
= & \frac{a}{k}\left(c\left(t_{2}-s\right)^{2}+2 d\left(t_{2}-s\right)\right)-1 \\
= & \frac{1}{k}\left(a c\left(t_{2}-s\right)^{2}+2 a d\left(t_{2}-s\right)-k\right) \\
\leq & 0
\end{aligned}
$$


guarantee that $x_{2}(t, s)$ is nonincreasing and concave in $t$ on $\left[\eta, t_{2}\right]$; we have $G(\eta, s) \geq G(t, s)$ for each $(t, s) \in\left[\eta, t_{2}\right] \times\left[\eta, t_{2}\right]$ :

$$
\begin{aligned}
G & \left(t_{2}, s\right) \\
= & x_{2}\left(t_{2}, s\right) \\
= & \frac{1}{k}\left(b+\frac{a}{2}\left(t_{2}-t_{1}\right)^{2}-a\left(\eta-t_{1}\right)\left(t_{2}-t_{1}\right)\right) \\
& \times\left(c\left(t_{2}-s\right)^{2}+2 d\left(t_{2}-s\right)\right) \\
& -\frac{1}{2}\left(t_{2}-s\right)^{2} \\
= & \frac{1}{2 k}\left(2 b c+a c\left(t_{2}-t_{1}\right)\left(t_{1}+t_{2}-2 \eta\right)-k\right)\left(t_{2}-s\right)^{2} \\
& +\frac{d}{k}\left(2 b+a\left(t_{2}-t_{1}\right)\left(t_{1}+t_{2}-2 \eta\right)\right)\left(t_{2}-s\right) \\
\geq & \frac{a d\left(t_{2}-s\right)}{k}(s-\eta)\left(t_{2}-\eta\right) \\
\geq & 0, \quad \frac{\partial}{\partial t} G\left(t_{2}, s\right)<0 . \\
\frac{\partial t}{\partial t} G(t, s)<0, & \frac{\partial}{\partial t} G(s, s)>0,
\end{aligned}
$$

From the above argument, we obtain that

$$
G(t, s) \geq 0
$$

$$
G(t, s) \geq \min \left\{\min _{s \in\left[t_{1}, \eta\right]} \frac{x_{1}\left(t_{1}, t_{1}\right)}{x_{1}(\eta, s)}, \min _{s \in\left[\eta, t_{2}\right]} \frac{x_{2}\left(t_{2}, s\right)}{x_{2}(\eta, s)}\right\} G(\eta, s)
$$

$$
\left|\frac{\partial}{\partial t} G(t, s)\right| \geq \min _{s \in\left[\eta, t_{2}\right]}\left|\frac{(\partial / \partial t) G(s, s)}{(\partial / \partial t) G\left(t_{2}, s\right)}\right| \frac{\partial}{\partial t} G(\eta, s)
$$

we have (29), since $(\partial / \partial t) G(t, s) \geq \min _{s \in\left[t_{1}, \eta\right]}((\partial / \partial t) G(s, s) /$ $\left.(\partial / \partial t) G\left(t_{1}, s\right)\right)(\partial / \partial t) G(\eta, s)=0$.

Case 2. For $t \leq s$,

$$
\begin{aligned}
& \frac{\partial}{\partial t} G(t, s) \\
& =\frac{\partial}{\partial t} y_{1}(t, s) \\
& =\frac{a}{k}(t-\eta)\left(c\left(t_{2}-s\right)^{2}+2 d\left(t_{2}-s\right)\right. \\
& \left.\quad-2\left(c\left(t_{2}-t_{1}\right)+d\right)(\eta-s)\right) \\
& \quad+(\eta-s) \quad
\end{aligned}
$$

$$
\begin{aligned}
= & \frac{t-\eta}{k} \\
& \times\left(a c\left(t_{2}-s\right)^{2}+2 a d\left(t_{2}-s\right)\right. \\
& \left.\quad-2 a\left(c\left(t_{2}-t_{1}\right)+d\right)(\eta-s)\right)+(\eta-s) \\
\leq & t-s \\
\leq & 0, \quad \frac{\partial^{2}}{\partial t^{2}} G(t, s)=\frac{\partial^{2}}{\partial t^{2}} y_{1}(t, s) \\
= & \frac{a}{k}\left(c\left(t_{2}-s\right)^{2}+2 d\left(t_{2}-s\right)\right. \\
\geq & \left.0 \quad-2\left(c\left(t_{2}-t_{1}\right)+d\right)(\eta-s)\right)
\end{aligned}
$$

imply that $y_{1}(t, s)$ is nonincreasing and convex in $t$ on $\left[t_{1}, \eta\right]$; it follows that $G(t, s) \geq G(\eta, s)$ for each $(t, s) \in\left[t_{1}, \eta\right] \times\left[t_{1}, \eta\right]$ :

$$
\begin{aligned}
& G(\eta, s)= y_{1}(\eta, s) \\
&= \frac{1}{k}\left(b-\frac{a}{2}\left(\eta-t_{1}\right)^{2}\right) \\
& \times\left(c\left(t_{2}-s\right)^{2}+2 d\left(t_{2}-s\right)\right. \\
& \geq\left.0, \quad-2\left(c\left(t_{2}-t_{1}\right)+d\right)(\eta-s)\right)+\left(\eta-t_{1}\right)(\eta-s) \\
& \frac{\partial}{\partial t} G(t, s)<0, \quad \frac{\partial}{\partial t} G(s, s)>0, \quad \frac{\partial}{\partial t} G\left(t_{1}, s\right)<0 .
\end{aligned}
$$

Likewise,

$$
\begin{aligned}
\frac{\partial}{\partial t} G(t, s) & =\frac{\partial}{\partial t} y_{2}(t, s) \\
& =\frac{a}{k}(t-\eta)\left(c\left(t_{2}-s\right)^{2}+2 d\left(t_{2}-s\right)\right) \\
& \geq 0 \\
\frac{\partial^{2}}{\partial t^{2}} G(t, s) & =\frac{\partial^{2}}{\partial t^{2}} y_{2}(t, s) \\
& =\frac{a}{k}\left(c\left(t_{2}-s\right)^{2}+2 d\left(t_{2}-s\right)\right) \\
& \geq 0
\end{aligned}
$$


guarantee that $y_{2}(t, s)$ is nondecreasing and convex in $t$ on $\left[\eta, t_{2}\right]$; it follows that $G(t, s) \geq G(\eta, s)$ for each $(t, s) \in\left[\eta, t_{2}\right] \times$ $\left[\eta, t_{2}\right]:$

$$
\begin{aligned}
& G(\eta, s)= y_{2}(\eta, s) \\
&= \frac{1}{k}\left(b-\frac{a}{2}\left(\eta-t_{1}\right)^{2}\right) \\
& \times\left(c\left(t_{2}-s\right)^{2}+2 d\left(t_{2}-s\right)\right) \\
& \geq 0, \\
& \frac{\partial}{\partial t} G(t, s)>0, \quad \frac{\partial}{\partial t} G(s, s)>0, \quad \frac{\partial}{\partial t} G\left(t_{2}, s\right)>0 .
\end{aligned}
$$

So we get

$$
G(t, s) \geq 0
$$

$$
G(t, s) \geq \min \left\{\min _{s \in\left[t_{1}, \eta\right]} \frac{y_{1}(\eta, \eta)}{y_{1}\left(t_{1}, s\right)}, \min _{s \in\left[\eta, t_{2}\right]} \frac{y_{2}(\eta, s)}{y_{2}\left(t_{2}, s\right)}\right\} G(\eta, s),
$$

$$
\left|\frac{\partial}{\partial t} G(t, s)\right| \geq \min _{s \in\left[t_{1}, \eta\right]}\left|\frac{(\partial / \partial t) G(s, s)}{(\partial / \partial t) G\left(t_{1}, s\right)}\right| \frac{\partial}{\partial t} G(\eta, s),
$$

we have (35), since $(\partial / \partial t) G(t, s) \geq \min _{s \in\left[\eta, t_{2}\right]}(((\partial / \partial t) G(s, s)) /$ $\left.\left((\partial / \partial t) G\left(t_{2}, s\right)\right)\right)(\partial / \partial t) G(\eta, s)=0$.

It is easy to show that $\left.\tau_{1}, \tau_{2} \in\right] 0,1[$. Therefore, we obtain that $G(t, s) \geq 0$ for each $(t, s) \in\left[t_{1}, t_{2}\right] \times\left[t_{1}, t_{2}\right] ;(27)-(36)$ imply the desired conclusion.

\section{A Priori Estimates}

Lemma 5. Let $\left(H_{1}\right),\left(H_{2}\right)$ hold. If $g \in C\left[t_{1}, t_{2}\right]$ with $g \geq 0$, then a solution of the problem

$$
\begin{gathered}
u^{\prime \prime \prime}(t)=g(t), \quad 0<t_{1}<t<t_{2}, \\
a u\left(t_{1}\right)-b u^{\prime \prime}\left(t_{1}\right)=0, \\
u^{\prime}(\eta)=0, \quad t_{1}<\eta<t_{2}, \\
c u\left(t_{2}\right)+d u^{\prime}\left(t_{2}\right)=0
\end{gathered}
$$

satisfies

$$
\begin{gathered}
u(t) \geq 0, \quad t \in\left[t_{1}, t_{2}\right], \\
\min _{t \in[\xi, \omega]} u(t) \geq \tau_{1} \max _{t \in\left[t_{1}, t_{2}\right]}|u(t)|,
\end{gathered}
$$

where $[\xi, \omega] \subseteq\left[t_{1}, t_{2}\right],(\xi<\omega)$.

Proof. A solution of problem (37) is $u(t)=\int_{t_{1}}^{t_{2}} G(t, s) g(s) d s$, where $G(t, s)$ is defined by (12); it is not difficult to show that the results hold by Lemma 4 and we omit the proof process.
Lemma 6. Let $\left(H_{1}\right),\left(H_{2}\right)$ hold. If $g \in C\left[t_{1}, t_{2}\right]$ with $g \geq 0$, then a solution of problem (37) satisfies

$$
\begin{gathered}
\max _{t \in\left[t_{1}, t_{2}\right]} u(t) \leq \tau_{3} \max _{t \in\left[t_{1}, t_{2}\right]}\left|u^{\prime}(t)\right|, \\
\max _{t \in\left[t_{1}, t_{2}\right]}\left|u^{\prime}(t)\right| \leq \tau_{4} \max _{t \in\left[t_{1}, t_{2}\right]}\left|u^{\prime \prime}(t)\right|,
\end{gathered}
$$

where

$$
\begin{aligned}
& \tau_{3} \\
& =\max \left\{\left(\left(b-\frac{a}{2}\left(\eta-t_{1}\right)^{2}\right)\left(c\left(t_{2}-t_{1}\right)^{2}+2 d\left(t_{2}-t_{1}\right)\right)\right.\right. \\
& \left.+k\left(\eta-t_{1}\right)^{2}\right) \\
& \times\left(\left(\eta-t_{1}\right)\left(k-a\left(c\left(t_{2}-t_{1}\right)^{2}+2 d\left(t_{2}-t_{1}\right)\right)\right)\right)^{-1}, \\
& \frac{\left(b-(a / 2)\left(\eta-t_{1}\right)^{2}\right)\left(c\left(t_{2}-\eta\right)^{2}+2 d\left(t_{2}-\eta\right)\right)}{\left(t_{2}-\eta\right)\left(k-a\left(c\left(t_{2}-\eta\right)^{2}+2 d\left(t_{2}-\eta\right)\right)\right)}, \\
& \left(b\left(c\left(t_{2}-t_{1}\right)^{2}+2 d\left(t_{2}-t_{1}\right)\right)\right) \\
& \times\left(\operatorname { i n f } _ { s \in [ t _ { 1 } , \eta ] } \left(a\left(\eta-t_{1}\right)\right.\right. \\
& \times\left(c\left(t_{2}-s\right)^{2}+2 d\left(t_{2}-s\right)\right. \\
& \left.-2\left(c\left(t_{2}-t_{1}\right)+d\right)(\eta-s)\right) \\
& +k(s-\eta)))^{-1} \\
& \left.\frac{b+(a / 2)\left(t_{2}-t_{1}\right)^{2}}{a\left(t_{2}-\eta\right)}\right\} \\
& \tau_{4}=t_{2}-\eta
\end{aligned}
$$

Proof. According to the nonnegative solution $u$ of problem (37), we establish a priori estimates as follows. For $t_{1} \leq$ $s \leq t \leq \eta$, we can check that $\max _{t \in\left[t_{1}, \eta\right]} u(t)=x_{1}(\eta, s)$, $\max _{t \in\left[t_{1}, \eta\right]}\left|u^{\prime}(t)\right|=(\partial / \partial t) x_{1}\left(t_{1}, s\right), \max _{t \in\left[t_{1}, \eta\right]}\left|u^{\prime \prime}(t)\right|=$ $-\left(\partial^{2} / \partial t^{2}\right) x_{1}(\eta, s)$; note that $(a / k)\left(c\left(t_{2}-s\right)^{2}+2 d\left(t_{2}-s\right)-\right.$ $\left.2\left(c\left(t_{2}-t_{1}\right)+d\right)(\eta-s)\right)-1 \leq 0$, so

$$
\begin{aligned}
& \frac{\max _{t \in\left[t_{1}, \eta\right]} u(t)}{\max _{t \in\left[t_{1}, \eta\right]}\left|u^{\prime}(t)\right|} \\
& \leq\left(\left(b-\frac{a}{2}\left(\eta-t_{1}\right)^{2}\right)\right. \\
& \quad \times\left(c\left(t_{2}-s\right)^{2}+2 d\left(t_{2}-s\right)-2\left(c\left(t_{2}-t_{1}\right)+d\right)(\eta-s)\right) \\
& \left.\quad+k\left(\eta-t_{1}\right)^{2}\right)
\end{aligned}
$$




$$
\begin{aligned}
& \times\left(( \eta - t _ { 1 } ) \left(k-a\left(c\left(t_{2}-s\right)^{2}+2 d\left(t_{2}-s\right)\right.\right.\right. \\
& \left.\left.\left.\quad-2\left(c\left(t_{2}-t_{1}\right)+d\right)(\eta-s)\right)\right)\right)^{-1} \\
& \leq\left(\left(b-\frac{a}{2}\left(\eta-t_{1}\right)^{2}\right)\right. \\
& \left.\quad \times\left(c\left(t_{2}-t_{1}\right)^{2}+2 d\left(t_{2}-t_{1}\right)\right)+k\left(\eta-t_{1}\right)^{2}\right) \\
& \times\left(\left(\eta-t_{1}\right)\left(k-a\left(c\left(t_{2}-t_{1}\right)^{2}+2 d\left(t_{2}-t_{1}\right)\right)\right)\right)^{-1}, \\
& \max _{t \in\left[t_{1}, \eta\right]} u^{\prime}(t) \\
& \max _{t \in\left[t_{1}, \eta\right]}\left|u^{\prime \prime}(t)\right| \\
& \leq\left(( \eta - t ) \left(k-a\left(c\left(t_{2}-s\right)^{2}+2 d\left(t_{2}-s\right)\right.\right.\right. \\
& \left.\left.\left.\quad-2\left(c\left(t_{2}-t_{1}\right)+d\right)(\eta-s)\right)\right)\right) \\
& \quad \times\left(k-a\left(c\left(t_{2}-s\right)^{2}+2 d\left(t_{2}-s\right)\right.\right. \\
& \left.\left.\quad-2\left(c\left(t_{2}-t_{1}\right)+d\right)(\eta-s)\right)\right)^{-1} \\
& \leq \eta-t_{1} . \quad
\end{aligned}
$$

For $\eta \leq s \leq t \leq t_{2}$, we can check that $\max _{t \in\left[\eta, t_{2}\right]} u(t)=$ $x_{2}(\eta, s), \max _{t \in\left[\eta, t_{2}\right]}\left|u^{\prime}(t)\right|=-(\partial / \partial t) x_{2}\left(t_{2}, s\right)$, $\max _{t \in\left[\eta, t_{2}\right]}\left|u^{\prime \prime}(t)\right|=-\left(\partial^{2} / \partial t^{2}\right) x_{2}\left(t_{2}, s\right)$; notice that $(a / k)$ $\left(c\left(t_{2}-s\right)^{2}+2 d\left(t_{2}-s\right)\right)-1 \leq 0$ and thus

$$
\begin{aligned}
& \frac{\max _{t \in\left[\eta, t_{2}\right]} u(t)}{\max _{t \in\left[\eta, t_{2}\right]}\left|u^{\prime}(t)\right|} \\
& \leq \frac{\left(b-(a / 2)\left(\eta-t_{1}\right)^{2}\right)\left(c\left(t_{2}-\eta\right)^{2}+2 d\left(t_{2}-\eta\right)\right)}{\left(t_{2}-\eta\right)\left(k-a\left(c\left(t_{2}-s\right)^{2}+2 d\left(t_{2}-s\right)\right)\right)} \\
& \leq \frac{\left(b-(a / 2)\left(\eta-t_{1}\right)^{2}\right)\left(c\left(t_{2}-\eta\right)^{2}+2 d\left(t_{2}-\eta\right)\right)}{\left(t_{2}-\eta\right)\left(k-a\left(c\left(t_{2}-\eta\right)^{2}+2 d\left(t_{2}-\eta\right)\right)\right)} \\
& \frac{\max _{t \in\left[\eta, t_{2}\right]} u^{\prime}(t)}{\max _{t \in\left[\eta, t_{2}\right]}\left|u^{\prime \prime}(t)\right|} \\
& \leq \frac{k(t-\eta)-a(t-\eta)\left(c\left(t_{2}-s\right)^{2}+2 d\left(t_{2}-s\right)\right)}{k-a\left(c\left(t_{2}-s\right)^{2}+2 d\left(t_{2}-s\right)\right)} \\
& \leq t_{2}-\eta .
\end{aligned}
$$

For $t_{1} \leq t \leq s \leq \eta$, we can check that $\max _{t \in\left[t_{1}, \eta\right]} u(t)=$ $y_{1}\left(t_{1}, s\right), \max _{t \in\left[t_{1}, \eta\right]}\left|u^{\prime}(t)\right|=\quad=\quad-(\partial / \partial t) y_{1}\left(t_{1}, s\right)$, $\max _{t \in\left[t_{1}, \eta\right]}\left|u^{\prime \prime}(t)\right|=\left(\partial^{2} / \partial t^{2}\right) y_{1}\left(t_{1}, s\right)$; note that $(a / k)$ $\left(c\left(t_{2}-s\right)^{2}+2 d\left(t_{2}-s\right)-2\left(c\left(t_{2}-t_{1}\right)+d\right)(\eta-s)\right)-1 \leq 0$ and so

$$
\begin{aligned}
& \frac{\max _{t \in\left[t_{1}, \eta\right]} u(t)}{\max _{t \in\left[t_{1}, \eta\right]}\left|u^{\prime(t)}\right|} \\
& \leq\left(b\left(c\left(t_{2}-t_{1}\right)^{2}+2 d\left(t_{2}-t_{1}\right)\right)\right) \\
& \quad \times\left(a ( \eta - t _ { 1 } ) \left(c\left(t_{2}-s\right)^{2}+2 d\left(t_{2}-s\right)\right.\right. \\
& \left.\left.\quad-2\left(c\left(t_{2}-t_{1}\right)+d\right)(\eta-s)\right)+k(s-\eta)\right)^{-1} \\
& \leq\left(b\left(c\left(t_{2}-t_{1}\right)^{2}+2 d\left(t_{2}-t_{1}\right)\right)\right) \\
& \times\left(\operatorname { i n f } _ { s \in t _ { 1 } , \eta ] } \left(a\left(\eta-t_{1}\right)\right.\right. \\
& \times\left(c\left(t_{2}-s\right)^{2}+2 d\left(t_{2}-s\right)\right. \\
& \left.-2\left(c\left(t_{2}-t_{1}\right)+d\right)(\eta-s)\right) \\
& +k(s-\eta)))^{-1}
\end{aligned}
$$

$$
\begin{aligned}
& \frac{\max _{t \in\left[t_{1}, \eta\right]} u^{\prime}(t)}{\max _{t \in\left[t_{1}, \eta\right]}\left|u^{\prime \prime}(t)\right|} \\
& \leq\left(a ( \eta - t _ { 1 } ) \left(c\left(t_{2}-s\right)^{2}+2 d\left(t_{2}-s\right)\right.\right. \\
& \left.\left.\quad-2\left(c\left(t_{2}-t_{1}\right)+d\right)(\eta-s)\right)+k(s-\eta)\right) \\
& \quad \times\left(a \left(c\left(t_{2}-s\right)^{2}+2 d\left(t_{2}-s\right)\right.\right. \\
& \left.\left.\quad-2\left(c\left(t_{2}-t_{1}\right)+d\right)(\eta-s)\right)\right)^{-1} \\
& \leq \eta-t_{1} .
\end{aligned}
$$

For $\eta \leq t \leq s \leq t_{2}$, we can check that $\max _{t \in\left[\eta, t_{2}\right]} u(t)=$ $y_{2}\left(t_{2}, s\right), \max _{t \in\left[\eta, t_{2}\right]}\left|u^{\prime}(t)\right|=(\partial / \partial t) y_{2}\left(t_{2}, s\right), \max _{t \in\left[\eta, t_{2}\right]}$ $\left|u^{\prime \prime}(t)\right|=\left(\partial^{2} / \partial t^{2}\right) y_{2}(\eta, s)$; then

$$
\begin{aligned}
& \frac{\max _{t \in\left[\eta, t_{2}\right]} u(t)}{\max _{t \in\left[\eta, t_{2}\right]}\left|u^{\prime}(t)\right|} \\
& =\frac{b+(a / 2)\left(t_{2}-t_{1}\right)^{2}-a\left(\eta-t_{1}\right)\left(t_{2}-t_{1}\right)}{a\left(t_{2}-\eta\right)} \\
& \leq \frac{b+(a / 2)\left(t_{2}-t_{1}\right)^{2}}{a\left(t_{2}-\eta\right)}
\end{aligned}
$$




$$
\begin{aligned}
& \frac{\max _{t \in\left[\eta, t_{2}\right]} u^{\prime}(t)}{\max _{t \in\left[\eta, t_{2}\right]}\left|u^{\prime \prime}(t)\right|} \\
& \leq \frac{a\left(t_{2}-\eta\right)\left(c\left(t_{2}-s\right)^{2}+2 d\left(t_{2}-s\right)\right)}{a\left(c\left(t_{2}-s\right)^{2}+2 d\left(t_{2}-s\right)\right)} \\
& =t_{2}-\eta .
\end{aligned}
$$

From the above argument, we can obtain the desired result (39).

Let $E$ be the Banach space $C^{2}\left[t_{1}, t_{2}\right]$ with the norm $\|u\|=$ $\max \left\{\|u\|_{0},\left\|u^{\prime}\right\|_{0},\left\|u^{\prime \prime}\right\|_{0}\right\}$, where $\|\cdot\|_{0}=\max _{t \in\left[t_{1}, t_{2}\right]}|\cdot(t)|$. Set a cone in $E$

$$
K=\left\{\begin{array}{c}
u \in E: u \text { is nonnegative on }\left[t_{1}, t_{2}\right], \text { and satisfies } a u\left(t_{1}\right)-b u^{\prime \prime}\left(t_{1}\right)=0, \\
u^{\prime}(\eta)=0, c u\left(t_{2}\right)+d u^{\prime}\left(t_{2}\right)=0, \min _{t \in[\xi, \omega]} u(t) \geq \tau_{1} \max _{t \in\left[t_{1}, t_{2}\right]}|u(t)|
\end{array}\right\}
$$

In view of the assumptions $\left(H_{1}\right)$ and $\left(H_{2}\right)$, take

$$
\begin{aligned}
u_{0}(t)= & -\bar{m} t^{3}+\frac{\lambda_{2}}{\lambda_{1}} \bar{m} t^{2}+\frac{\lambda_{3}}{\lambda_{1}} \bar{m} t \\
& +\left(t_{1}^{3}-\frac{6 b}{a} t_{1}+\left(\frac{2 b}{a}-t_{1}^{2}\right) \frac{\lambda_{2}}{\lambda_{1}}-t_{1} \frac{\lambda_{3}}{\lambda_{1}}\right) \bar{m},
\end{aligned}
$$

where $\lambda_{1}=2 \eta\left(a c t_{1}-a c t_{2}-a d\right)-a c t_{1}^{2}+2 b c+a c t_{2}^{2}+2 a d t_{2}$, $\lambda_{2}=3 \eta^{2}\left(a c t_{1}-a c t_{2}-a d\right)-a c t_{1}^{3}+6 b c t_{1}+a c t_{2}^{3}+3 a d t_{2}^{2}, \lambda_{3}=$ $3 \eta^{2}\left(-a c t_{1}^{2}+a c t_{2}^{2}+2 a d t_{2}\right)+2 \eta\left(a c t_{1}^{3}-6 b c t_{1}-a c t_{2}^{3}-3 a d t_{2}^{2}\right)$, and $\bar{m}$ is an arbitrary positive constant. By some simplifying, we obtain that $\lambda_{1}, \lambda_{2} \geq 0$. Set a constant

$$
\begin{array}{r}
\vartheta:=\max \{- \\
-t_{1}^{3}+\frac{\lambda_{2}}{\lambda_{1}} t_{1}^{2}+\frac{\lambda_{3}}{\lambda_{1}} t_{1}+\lambda_{4}, \\
-\eta^{3}+\frac{\lambda_{2}}{\lambda_{1}} \eta^{2}+\frac{\lambda_{3}}{\lambda_{1}} \eta+\lambda_{4}, \\
-\bar{t}^{3}+\frac{\lambda_{2}}{\lambda_{1}} \bar{t}^{2}+\frac{\lambda_{3}}{\lambda_{1}} \bar{t}+\lambda_{4}, \\
\left.-t_{2}^{3}+\frac{\lambda_{2}}{\lambda_{1}} t_{2}^{2}+\frac{\lambda_{3}}{\lambda_{1}} t_{2}+\lambda_{4}\right\},
\end{array}
$$

where $\bar{t}=\left(\lambda_{2}+\sqrt{3 \lambda_{1} \lambda_{3}+\lambda_{2}^{2}}\right) / 3 \lambda_{1}, \quad \lambda_{4}=t_{1}^{3}-(6 b / a) t_{1}+$ $\left((2 b / a)-t_{1}^{2}\right)\left(\lambda_{2} / \lambda_{1}\right)-t_{1}\left(\lambda_{3} / \lambda_{1}\right)$. It is not difficult to check that $u_{0}(t)$ is nonnegative on $\left[t_{1}, t_{2}\right]$ and satisfies $a u_{0}\left(t_{1}\right)-b u_{0}^{\prime \prime}\left(t_{1}\right)=$ $0, u_{0}^{\prime}(\eta)=0, c u_{0}\left(t_{2}\right)+d u_{0}^{\prime}\left(t_{2}\right)=0$. Under the assumption $\min \left\{-\xi^{3}+\left(\lambda_{2} / \lambda_{1}\right) \xi^{2}+\left(\lambda_{3} / \lambda_{1}\right) \xi+\lambda_{4},-\omega^{3}+\left(\lambda_{2} / \lambda_{1}\right) \omega^{2}+\right.$ $\left.\left(\lambda_{3} / \lambda_{1}\right) \omega+\lambda_{4},-\eta^{3}+\left(\lambda_{2} / \lambda_{1}\right) \eta^{2}+\left(\lambda_{3} / \lambda_{1}\right) \eta+\lambda_{4}\right\} \geq \tau_{1} \vartheta$, we have $\min _{t \in[\xi, \omega]} u_{0}(t) \geq \tau_{1} \max _{t \in\left[t_{1}, t_{2}\right]}\left|u_{0}(t)\right|$; that is, $u_{0} \in K$, so $K \backslash\{0\} \neq \emptyset$. By assumption $\left(H_{3}\right)$, both $0<\int_{t_{1}}^{t_{2}} G\left(t_{1}, s\right) h(s) d s<$ $\infty, 0<\int_{t_{1}}^{t_{2}} G(\eta, s) h(s) d s<\infty$ and $0<\int_{t_{1}}^{t_{2}} G\left(t_{2}, s\right) h(s) d s<$ $\infty$ hold. Thus, we can define an operator $T$ by

$$
\begin{array}{r}
(T u)(t):=\int_{t_{1}}^{t_{2}} G(t, s) h(s) f\left(s, u(s), u^{\prime}(s), u^{\prime \prime}(s)\right) d s, \\
t \in\left[t_{1}, t_{2}\right] .
\end{array}
$$

Lemma 7. Let $\left(H_{1}\right)-\left(H_{4}\right)$ hold. Then, $T: K \rightarrow K$ is completely continuous.

Proof. Since conditions $\left(H_{1}\right)-\left(H_{4}\right)$ hold, by Lemma 5, we observe that $(T u)(t) \geq 0$ for $t \in\left[t_{1}, t_{2}\right] ; a(T u)\left(t_{1}\right)-$ $b(T u)^{\prime \prime}\left(t_{1}\right)=0,(T u)^{\prime}(\eta)=0, c(T u)\left(t_{2}\right)+d(T u)^{\prime}\left(t_{2}\right)=$ $0, \min _{t \in[\xi, \omega]}(T u)(t) \geq \tau_{1} \max _{t \in\left[t_{1}, t_{2}\right]}|(T u)(t)|$ are satisfied, so $T u \in K$. Since $h(t)$ may be singular at $t=t_{1}$ and/or $t=t_{2}$, we will take the arguments to show that the operator $T$ is completely continuous. Assume that $u_{n}, u_{0} \in K(n=1,2, \ldots)$ satisfy $\left\|u_{n}-u_{0}\right\| \rightarrow 0(n \rightarrow \infty)$; then there exists $R>0$ such that $\left\|u_{n}\right\| \leq R$ for arbitrary positive integer $n$. One has that

$$
\begin{aligned}
& \left\|T u_{n}-T u_{0}\right\|_{0} \\
& =\max _{t \in\left[t_{1}, t_{2}\right]}\left|T u_{n}(t)-T u_{0}(t)\right| \\
& \leq \max _{t \in\left[t_{1}, \eta\right]} \mid \int_{t_{1}}^{t_{2}} G(t, s) h(s) \\
& \times\left(f\left(s, u_{n}, u_{n}^{\prime}, u_{n}^{\prime \prime}\right)-f\left(s, u_{0}, u_{0}^{\prime}, u_{0}^{\prime \prime}\right)\right) d s \mid \\
& +\max _{t \in\left[\eta, t_{2}\right]} \mid \int_{t_{1}}^{t_{2}} G(t, s) h(s) \\
& \quad \times\left(f\left(s, u_{n}, u_{n}^{\prime}, u_{n}^{\prime \prime}\right)-f\left(s, u_{0}, u_{0}^{\prime}, u_{0}^{\prime \prime}\right)\right) d s \mid \\
& \leq \max _{t \in\left[t_{1}, \eta\right]} \mid \int_{t_{1}}^{t} G(\eta, s) h(s) \\
& \quad \times\left(f\left(s, u_{n}, u_{n}^{\prime}, u_{n}^{\prime \prime}\right)-f\left(s, u_{0}, u_{0}^{\prime}, u_{0}^{\prime \prime}\right)\right) d s \\
& +\int_{t}^{\eta} G\left(t_{1}, s\right) h(s) \\
& \times\left(f\left(s, u_{n}, u_{n}^{\prime}, u_{n}^{\prime \prime}\right)-f\left(s, u_{0}, u_{0}^{\prime}, u_{0}^{\prime \prime}\right)\right) d s \mid
\end{aligned}
$$




$$
\begin{aligned}
& +\max _{t \in\left[\eta, t_{2}\right]} \mid \int_{\eta}^{t} G(\eta, s) h(s) \\
& \times\left(f\left(s, u_{n}, u_{n}^{\prime}, u_{n}^{\prime \prime}\right)-f\left(s, u_{0}, u_{0}^{\prime}, u_{0}^{\prime \prime}\right)\right) d s \\
& +\int_{t}^{t_{2}} G\left(t_{2}, s\right) h(s) \\
& \times\left(f\left(s, u_{n}, u_{n}^{\prime}, u_{n}^{\prime \prime}\right)\right. \\
& \left.-f\left(s, u_{0}, u_{0}^{\prime}, u_{0}^{\prime \prime}\right)\right) d s \\
& \leq \int_{t_{1}}^{\eta} G(\eta, s) h(s) \\
& \times\left|f\left(s, u_{n}, u_{n}^{\prime}, u_{n}^{\prime \prime}\right)-f\left(s, u_{0}, u_{0}^{\prime}, u_{0}^{\prime \prime}\right)\right| d s \\
& +\int_{t_{1}}^{\eta} G\left(t_{1}, s\right) h(s) \\
& \times\left|f\left(s, u_{n}, u_{n}^{\prime}, u_{n}^{\prime \prime}\right)-f\left(s, u_{0}, u_{0}^{\prime}, u_{0}^{\prime \prime}\right)\right| d s \\
& +\int_{\eta}^{t_{2}} G(\eta, s) h(s) \\
& \times\left|f\left(s, u_{n}, u_{n}^{\prime}, u_{n}^{\prime \prime}\right)-f\left(s, u_{0}, u_{0}^{\prime}, u_{0}^{\prime \prime}\right)\right| d s \\
& +\int_{\eta}^{t_{2}} G\left(t_{2}, s\right) h(s) \\
& \times\left|f\left(s, u_{n}, u_{n}^{\prime}, u_{n}^{\prime \prime}\right)-f\left(s, u_{0}, u_{0}^{\prime}, u_{0}^{\prime \prime}\right)\right| d s, \\
& \left\|\left(T u_{n}\right)^{\prime}-\left(T u_{0}\right)^{\prime}\right\|_{0} \\
& =\max _{t \in\left[t_{1}, t_{2}\right]}\left|\left(T u_{n}\right)^{\prime}(t)-\left(T u_{0}\right)^{\prime}(t)\right| \\
& \leq \max _{t \in\left[t_{1}, \eta\right]} \mid \int_{t_{1}}^{t_{2}} \frac{\partial}{\partial t} G(t, s) h(s) \\
& \times\left|f\left(s, u_{n}, u_{n}^{\prime}, u_{n}^{\prime \prime}\right)-f\left(s, u_{0}, u_{0}^{\prime}, u_{0}^{\prime \prime}\right)\right| d s \mid \\
& +\max _{\left.t \in] \eta, t_{2}\right]} \mid \int_{t_{1}}^{t_{2}} \frac{\partial}{\partial t} G(t, s) h(s) \\
& \times\left|f\left(s, u_{n}, u_{n}^{\prime}, u_{n}^{\prime \prime}\right)-f\left(s, u_{0}, u_{0}^{\prime}, u_{0}^{\prime \prime}\right)\right| d s \mid \\
& \leq \max _{t \in\left[t_{1}, \eta\right]} \int_{t_{1}}^{t_{2}}\left|\frac{\partial}{\partial t} G(t, s)\right| h(s) \\
& \times\left|f\left(s, u_{n}, u_{n}^{\prime}, u_{n}^{\prime \prime}\right)-f\left(s, u_{0}, u_{0}^{\prime}, u_{0}^{\prime \prime}\right)\right| d s \\
& +\max _{\left.t \in] \eta, t_{2}\right]} \int_{t_{1}}^{t_{2}}\left|\frac{\partial}{\partial t} G(t, s)\right| h(s) \\
& \times\left|f\left(s, u_{n}, u_{n}^{\prime}, u_{n}^{\prime \prime}\right)-f\left(s, u_{0}, u_{0}^{\prime}, u_{0}^{\prime \prime}\right)\right| d s,
\end{aligned}
$$

$$
\begin{aligned}
& \left\|\left(T u_{n}\right)^{\prime \prime}-\left(T u_{0}\right)^{\prime \prime}\right\|_{0} \\
& =\max _{t \in\left[t_{1}, t_{2}\right]}\left|\left(T u_{n}\right)^{\prime \prime}(t)-\left(T u_{0}\right)^{\prime \prime}(t)\right| \\
& \leq \max _{t \in\left[t_{1}, \eta\right]} \mid \int_{t_{1}}^{t_{2}} \frac{\partial^{2}}{\partial t^{2}} G(t, s) h(s) \\
& \quad \times\left|f\left(s, u_{n}, u_{n}^{\prime}, u_{n}^{\prime \prime}\right)-f\left(s, u_{0}, u_{0}^{\prime}, u_{0}^{\prime \prime}\right)\right| d s \mid \\
& +\max _{\left.t \in] \eta, t_{2}\right]} \mid \int_{t_{1}}^{t_{2}} \frac{\partial^{2}}{\partial t^{2}} G(t, s) h(s) \\
& \leq \max _{t \in\left[t_{1}, \eta\right]} \int_{t_{1}}^{t_{2}}\left|\frac{\partial^{2}}{\partial t^{2}} G(t, s)\right| h(s) \\
& \times\left|f\left(s, u_{n}, u_{n}^{\prime}, u_{n}^{\prime \prime}\right)-f\left(s, u_{0}, u_{0}^{\prime}, u_{0}^{\prime \prime}\right)\right| d s \mid \\
& \quad+\max _{\left.t \in] \eta, t_{2}\right]} \int_{t_{1}}^{t_{2}}\left|\frac{\partial^{2}}{\partial t^{2}} G(t, s)\right| h(s) \\
& \left.\times\left|f\left(s, u_{n}, u_{n}^{\prime}, u_{n}^{\prime \prime}\right)-f\left(s, u_{0}, u_{0}^{\prime}, u_{0}^{\prime \prime}\right)\right| d s . u_{n}^{\prime \prime}\right)-f\left(s, u_{0}, u_{0}^{\prime}, u_{0}^{\prime \prime}\right) \mid d s
\end{aligned}
$$

In view of the continuity of $f(t, \mu, \nu, \omega)$ in $\mu, \nu, \omega$, we can obtain that, for any $t \in\left[t_{1}, t_{2}\right],\left\|T u_{n}-T u_{0}\right\|=$ $\max \left\{\left\|T_{n} u-T u\right\|_{0},\left\|\left(T_{n} u\right)^{\prime}-(T u)^{\prime}\right\|_{0},\left\|\left(T_{n}\right)^{\prime \prime} u-(T u)^{\prime \prime}\right\|_{0}\right\} \rightarrow$ $0(n \rightarrow \infty)$. This means that the operator $T: K \rightarrow K$ is continuous. It follows from the properties of the function $h$ that we can choose two sequences $\left\{\alpha_{n}\right\}_{n=1}^{\infty} \subset\left[t_{1}, \eta[\right.$, $\left.\left.\left\{\beta_{n}\right\}_{n=1}^{\infty} \subset\right] \eta, t_{2}\right]$. Obviously $\alpha_{n}<\beta_{n}$ for any $n \geq 1$ and $\alpha_{n} \rightarrow t_{1}, \beta_{n} \rightarrow t_{2}$ as $n \rightarrow \infty$, respectively. Define

$$
h_{n}(t)= \begin{cases}\inf \left\{h(t), h\left(\alpha_{n}\right)\right\}, & t \in\left[t_{1}, \alpha_{n}\right], \\ h(t), & t \in\left[\alpha_{n}, \beta_{n}\right], \\ \inf \left\{h\left(\beta_{n}\right), h(t)\right\}, & t \in\left[\beta_{n}, t_{2}\right],\end{cases}
$$

and an operator sequence $T_{n}$ by

$$
\begin{array}{r}
\left(T_{n} u\right)(t)=\int_{t_{1}}^{t_{2}} G(t, s) h_{n}(s) f\left(s, u(s), u^{\prime}(s), u^{\prime \prime}(s)\right) d s \\
t \in\left[t_{1}, t_{2}\right] .
\end{array}
$$

Clearly, $h_{n}(t):\left[t_{1}, t_{2}\right] \rightarrow[0, \infty[$ is a piecewise continuous function; the operator $T_{n}: K \rightarrow K$ is well defined. Further, we have that $T_{n}: K \rightarrow K$ is completely continuous.

Let $r>0, \mathbb{B}_{r}:=\left\{u \in K:\left\|u^{\prime \prime}\right\|_{0} \leq r\right\}$ and $M_{r}=$ $\max \left\{f(t, \mu, \nu, \omega): \mu \in K, t \in\left[t_{1}, t_{2}\right], \mu \in\left[0, \tau_{3} \tau_{4} r\right], \nu \in\right.$ $\left.\left[-\tau_{4} r, \tau_{4} r\right], \omega \in[-r, r]\right\}$. We will show that $T_{n}$ approaches $T$ uniformly on $\mathbb{B}_{r}$ as $n \rightarrow \infty$. From the absolute continuity of integral, we obtain

$$
\lim _{n \rightarrow \infty} \max _{t \in\left[t_{1}, t_{2}\right]} \int_{l(n)} G(t, s) h(s) d s=0,
$$


where $l(n)=\left[t_{1}, \alpha_{n}\right] \cup\left[\beta_{n}, t_{2}\right]$. Green's functions $G(t, s)$ and (52) imply that, for any $u \in \mathbb{B}_{r}, t \in\left[t_{1}, \alpha_{n}\right]$; we have

$$
\begin{aligned}
& \left\|T_{n} u-T u\right\|_{0} \\
& =\max _{t \in\left[t_{1}, \alpha_{n}\right]}\left|\int_{t_{1}}^{t_{2}} G(t, s)\left(h_{n}(s)-h(s)\right) f\left(s, u, u^{\prime}, u^{\prime \prime}\right) d s\right| \\
& \leq M_{r} \max _{t \in\left[t_{1}, \alpha_{n}\right]} \int_{t_{1}}^{t_{2}} G(t, s)\left|h_{n}(s)-h(s)\right| d s \\
& \leq M_{r} \max _{t \in\left[t_{1}, \alpha_{n}\right]} \int_{l(n)} G(t, s) h(s) d s \longrightarrow 0 \\
& (n \longrightarrow \infty) .
\end{aligned}
$$

For any $u \in \mathbb{B}_{r}, t \in\left[\beta_{n}, t_{2}\right]$, we get that $\left\|T_{n} u-T u\right\|_{0} \rightarrow$ $0(n \rightarrow \infty)$ and, for any $u \in \mathbb{B}_{r}, t \in\left[\alpha_{n}, \beta_{n}\right]$, there is $\left\|T_{n} u-T u\right\|_{0}=0(n \geq 1)$.

Similarly, for any $u \in \mathbb{B}_{r}, t \in\left[t_{1}, \alpha_{n}\right], t \in\left[\alpha_{n}, \beta_{n}\right]$, or $t \in\left[\beta_{n}, t_{2}\right]$, we can obtain that $\left\|\left(T_{n} u\right)^{\prime}-(T u)^{\prime}\right\|_{0} \rightarrow 0(n \rightarrow$ $\infty)$ and $\left\|\left(T_{n} u\right)^{\prime \prime}-(T u)^{\prime \prime}\right\|_{0} \rightarrow 0(n \rightarrow \infty)$, respectively.

The above argument implies that $\left\|T_{n} u-T u\right\| \rightarrow 0(n \rightarrow$ $\infty$ ); that is, the sequence $T_{n}$ uniformly approximates $T$ on any bounded subset of $K$. Hence, $T: K \rightarrow K$ is completely continuous.

\section{Single Solution}

Now, we are ready to present the sufficient conditions for the existence of at least one positive solution to the problem $\left(\mathscr{P}_{s}\right)$.

Theorem 8. Suppose $\left(H_{1}\right)-\left(H_{4}\right)$ hold; $f(t, 0,0,0) \not \equiv 0$ on any subinterval of $\left[t_{1}, t_{2}\right]$ and

$\left(C_{1}\right)$ there exist a constant $L$ and a function $F(t)$ which is integrable on $\left[t_{1}, t_{2}\right]$, satisfying

$$
\begin{array}{r}
f^{2}(t, \mu, \nu, \varpi) \leq F(t), \\
(t, \mu, v, \varpi) \in\left[t_{1}, t_{2}\right] \times\left[L, \infty[\times]-\infty, \infty\left[{ }^{2},\right.\right.
\end{array}
$$

$\left(C_{2}\right)$ there exist constants $m, M(m<M)$ such that $[m, M] \subseteq[\xi, \omega]$ and satisfy

$$
\lim _{\mu \rightarrow 0^{+}} \min _{t \in[m, M]} \frac{h f(t, \mu, \nu, \omega)}{\mu}=\infty .
$$

Then, the boundary value problem $\left(\mathscr{P}_{s}\right)$ has at least one positive solution.

Proof. By the definition of operator $T$ and Lemma 7, it suffices to show that the conditions of Lemma 1 hold with respect to $T$. Furthermore, from the fact that $G(t, s)$ is Green's function, it is not difficult to prove that a fixed point of $T$ is coincident with the solution of the boundary value problem $\left(\mathscr{P}_{s}\right)$, so we concentrate on the existence of the fixed point of the operator $T$.
First of all, we show that

$$
\lim _{v \rightarrow \infty} \frac{\Phi(v)}{v}=0,
$$

where

$$
\begin{aligned}
& \Phi(v) \\
& :=\sup _{u \in \partial \mathscr{K}(v)} \max \left\{\max _{t \in\left[t_{1}, t_{2}\right]} \mid \int_{t_{1}}^{t_{2}} G(t, s) h(s)\right. \\
& \times f\left(s, u, u^{\prime}, u^{\prime \prime}\right) d s \mid, \\
& \max _{t \in\left[t_{1}, t_{2}\right]} \mid \int_{t_{1}}^{t_{2}} \frac{\partial}{\partial t} G(t, s) h(s) \\
& \times f\left(s, u, u^{\prime}, u^{\prime \prime}\right) d s \mid, \\
& \max _{t \in\left[t_{1}, t_{2}\right]} \mid \int_{t_{1}}^{t_{2}} \frac{\partial^{2}}{\partial t^{2}} G(t, s) h(s) \\
& \left.\times f\left(s, u, u^{\prime}, u^{\prime \prime}\right) d s \mid\right\},
\end{aligned}
$$

and $\mathscr{K}(v)=\left\{u \in K:\|u\|_{0} \leq v, v \in\right] 0, \infty[\}$. For convenience, we introduce notation as follows:

$$
\mathscr{L}(t)=\left\{t \in\left[t_{1}, t_{2}\right]: u(t) \leq L\right\},
$$

$$
\begin{array}{r}
M_{1} \\
=\max \left\{\left(\int_{t_{1}}^{t_{2}}\left(\max \left\{G\left(t_{1}, s\right), G(\eta, s), G\left(t_{2}, s\right)\right\}\right)^{2}\right.\right. \\
\left.\times h^{2}(s) d s\right)^{1 / 2}, \\
\left(\int _ { t _ { 1 } } ^ { t _ { 2 } } \left(\operatorname { m a x } \left\{\left|\frac{\partial}{\partial t} G\left(t_{1}, s\right)\right|,\right.\right.\right. \\
\left.\left.\times \frac{\partial}{\partial t} G(\eta, s),\left|\frac{\partial}{\partial t} G\left(t_{2}, s\right)\right|\right\}\right)^{2} \\
\left(\int _ { t _ { 1 } } ^ { t _ { 2 } } \left(\operatorname { m a x } \left\{\left|\frac{\partial^{2}}{\partial t^{2}} G\left(t_{1}, s\right)\right|,\left|\frac{\partial^{2}}{\partial t^{2}} G(\eta, s)\right|,\right.\right.\right. \\
\left.\left.\times h^{1 / 2}(s) d s\right)^{1 / 2}\right\},
\end{array}
$$




$$
\begin{aligned}
& M_{2} \\
& =\max _{\left.\left(t, u, u^{\prime}\right) \in\left[t_{1}, t_{2}\right] \times[0, L] \times\right]-\infty, \infty\left[^{2}\right.} f^{2}\left(t, u(t), u^{\prime}(t), u^{\prime \prime}(t)\right), \\
& M_{3}=\int_{t_{1}}^{t_{2}} F(s) d s, \\
& M_{4}=\min \left\{\min _{s \in[m, M]} G(\eta, s), \min _{s \in[m, M]}\left|\frac{\partial^{2}}{\partial t^{2}} G(\eta, s)\right|\right\} .
\end{aligned}
$$

By the well-known Hölder's inequality, for any $t \in\left[t_{1}, t_{2}\right]$ and $u \in \partial \mathscr{K}(v)$, by using hypothesis $\left(C_{1}\right)$, we get

$$
\begin{aligned}
& \max _{t \in\left[t_{1}, t_{2}\right]}\left|\int_{t_{1}}^{t_{2}} G(t, s) h(s) f\left(s, u, u^{\prime}, u^{\prime \prime}\right) d s\right| \\
& \leq \int_{t_{1}}^{t_{2}} \max \left\{G\left(t_{1}, s\right), G(\eta, s), G\left(t_{2}, s\right)\right\} \\
& \times h(s) f\left(s, u, u^{\prime}, u^{\prime \prime}\right) d s \\
& \leq\left(\int_{t_{1}}^{t_{2}}\left(\max \left\{G\left(t_{1}, s\right), G(\eta, s), G\left(t_{2}, s\right)\right\}\right)^{2} h^{2}(s) d s\right)^{1 / 2} \\
& \times\left(\int_{t_{1}}^{t_{2}} f^{2}\left(s, u, u^{\prime}, u^{\prime \prime}\right) d s\right)^{1 / 2} \\
& \leq M_{1}\left(\int_{\left[t_{1}, t_{2}\right]-\mathscr{L}(t)} f^{2}\left(s, u, u^{\prime}, u^{\prime \prime}\right) d s\right. \\
& \left.+\int_{\mathscr{L}(t)} f^{2}\left(s, u, u^{\prime}\right) d s\right)^{1 / 2} \\
& \leq M_{1}\left(\int_{t_{1}}^{t_{2}} F(s) d s+\int_{\mathscr{L}(t)} f^{2}\left(s, u, u^{\prime}, u^{\prime \prime}\right) d s\right)^{1 / 2} \\
& \leq M_{1}\left(M_{2}\left(t_{2}-t_{1}\right)+M_{3}\right)^{1 / 2} \text {, } \\
& \max _{t \in\left[t_{1}, t_{2}\right]}\left|\int_{t_{1}}^{t_{2}} \frac{\partial}{\partial t} G(t, s) h(s) f\left(s, u, u^{\prime}, u^{\prime \prime}\right) d s\right| \\
& \leq \int_{t_{1}}^{t_{2}} \max \left\{\left|\frac{\partial}{\partial t} G\left(t_{1}, s\right)\right|, \frac{\partial}{\partial t} G(\eta, s),\left|\frac{\partial}{\partial t} G\left(t_{2}, s\right)\right|\right\} \\
& \times h(s) f\left(s, u, u^{\prime}, u^{\prime \prime}\right) d s \\
& \leq\left(\int_{t_{1}}^{t_{2}}\left(\max \left\{\left|\frac{\partial}{\partial t} G\left(t_{1}, s\right)\right|, \frac{\partial}{\partial t} G(\eta, s),\left|\frac{\partial}{\partial t} G\left(t_{2}, s\right)\right|\right\}\right)^{2}\right. \\
& \left.\times h^{2}(s) d s\right)^{1 / 2}\left(\int_{t_{1}}^{t_{2}} f^{2}\left(s, u, u^{\prime}, u^{\prime \prime}\right) d s\right)^{1 / 2}
\end{aligned}
$$

$$
\begin{aligned}
& \leq M_{1}\left(M_{2}\left(t_{2}-t_{1}\right)+M_{3}\right)^{1 / 2}, \\
& \max _{t \in\left[t_{1}, t_{2}\right]}\left|\int_{t_{1}}^{t_{2}} \frac{\partial^{2}}{\partial t^{2}} G(t, s) h(s) f\left(s, u, u^{\prime}, u^{\prime \prime}\right) d s\right| \\
& \leq \int_{t_{1}}^{t_{2}} \max \left\{\left|\frac{\partial^{2}}{\partial t^{2}} G\left(t_{1}, s\right)\right|,\left|\frac{\partial^{2}}{\partial t^{2}} G(\eta, s)\right|,\right. \\
& \leq\left(\int _ { t _ { 1 } } ^ { t _ { 2 } } \left(\max \left\{\left|\frac{\partial^{2}}{\partial t^{2}} G\left(t_{2}, s\right)\right|\right\} h(s) f\left(s, u, u^{\prime}, u^{\prime \prime}\right) d s\right.\right. \\
& \left.\quad \times h^{2}(s) d s\right)^{1 / 2}\left(\int_{t_{1}}^{t_{2}} f^{2}\left(s, u, u^{\prime}, u^{\prime \prime}\right) d s\right)^{1 / 2} \\
& \leq M_{1}\left(M_{2}\left(t_{2}-t_{1}\right)+M_{3}\right)^{1 / 2} .
\end{aligned}
$$

Consequently, $\lim _{v \rightarrow \infty}(\Phi(v) / v) \leq \lim _{v \rightarrow \infty}\left(M_{1}\left(M_{2}\left(t_{2}-\right.\right.\right.$ $\left.\left.\left.t_{1}\right)+M_{3}\right)^{1 / 2} / v\right)=0$.

Next, we verify that

$$
\lim _{v \rightarrow 0^{+}} \frac{\Psi(v)}{v}=\infty
$$

where

$\Psi(v)$

$$
\begin{gathered}
:=\inf _{u \in \partial \mathscr{K}(v)} \max \left\{\max _{t \in\left[t_{1}, t_{2}\right]}\left|\int_{t_{1}}^{t_{2}} G(t, s) h(s) f\left(s, u, u^{\prime}, u^{\prime \prime}\right) d s\right|,\right. \\
\max _{t \in\left[t_{1}, t_{2}\right]} \mid \int_{t_{1}}^{t_{2}} \frac{\partial}{\partial t} G(t, s) h(s) \\
\times f\left(s, u, u^{\prime}, u^{\prime \prime}\right) d s \mid \\
\max _{t \in\left[t_{1}, t_{2}\right]} \mid \int_{t_{1}}^{t_{2}} \frac{\partial^{2}}{\partial t^{2}} G(t, s) h(s) \\
\left.\times f\left(s, u, u^{\prime}, u^{\prime \prime}\right) d s \mid\right\} .
\end{gathered}
$$

In fact, hypothesis $\left(C_{2}\right)$ implies that, for any $u \in K, n \in \mathbb{N}$, there exists $\epsilon_{n}>0$ such that $\epsilon_{n} \rightarrow 0$ and

$$
\frac{h f(t, \mu, \nu, \omega)}{\mu} \geq n,
$$

$$
(t, \mu, \nu, \omega) \in[m, M] \times] 0, \epsilon_{n}[\times]-\infty, \infty\left[^{2} .\right.
$$


Then by (62) and Lemma 4, we have

$$
\begin{aligned}
& \max _{t \in\left[t_{1}, t_{2}\right]}\left|\int_{t_{1}}^{t_{2}} G(t, s) h(s) f\left(s, u, u^{\prime}, u^{\prime \prime}\right) d s\right| \\
& =\max _{t \in[\xi, \omega]} \int_{t_{1}}^{t_{2}} G(t, s) h(s) f\left(s, u, u^{\prime}, u^{\prime \prime}\right) d s \\
& \geq \tau_{1} \int_{t_{1}}^{t_{2}} G(\eta, s) h(s) f\left(s, u, u^{\prime}, u^{\prime \prime}\right) d s \\
& \geq \tau_{1} M_{4} \min _{t \in[m, M]} u(t) \int_{m}^{M} \frac{h(s) f\left(s, u, u^{\prime}, u^{\prime \prime}\right)}{u} d s \\
& \geq \tau_{1}^{2} M_{4}\|u\|_{0} \int_{m}^{M} \frac{h(s) f\left(s, u, u^{\prime}, u^{\prime \prime}\right)}{u} d s, \\
& \max _{t \in\left[t_{1}, t_{2}\right]}\left|\int_{t_{1}}^{t_{2}} \frac{\partial}{\partial t} G(t, s) h(s) f\left(s, u, u^{\prime}, u^{\prime \prime}\right) d s\right| \\
& =\max _{t \in\left[t_{1}, t_{2}\right]} \int_{t_{1}}^{t_{2}}\left|\frac{\partial}{\partial t} G(t, s)\right| h(s) f\left(s, u, u^{\prime}, u^{\prime \prime}\right) d s \\
& \geq \tau_{2} \int_{t_{1}}^{t_{2}} \frac{\partial}{\partial t} G(\eta, s) h(s) f\left(s, u, u^{\prime}, u^{\prime \prime}\right) d s \\
& \geq \tau_{2} M_{4} \min _{t \in[m, M]} u(t) \int_{m}^{M} \frac{h(s) f\left(s, u, u^{\prime}, u^{\prime \prime}\right)}{u} d s \\
& \geq \tau_{1} \tau_{2} M_{4}\|u\|_{0} \int_{m}^{M} \frac{h(s) f\left(s, u, u^{\prime}, u^{\prime \prime}\right)}{u} d s, \\
& \max _{t \in\left[t_{1}, t_{2}\right]}\left|\int_{t_{1}}^{t_{2}} \frac{\partial^{2}}{\partial t^{2}} G(t, s) h(s) f\left(s, u, u^{\prime}, u^{\prime \prime}\right) d s\right| \\
& =\max _{\left.t \in t_{1}, t_{2}\right]} \int_{t_{1}}^{t_{2}}\left|\frac{\partial^{2}}{\partial t^{2}} G(t, s)\right| h(s) f\left(s, u, u^{\prime}, u^{\prime \prime}\right) d s \\
& =\int_{t_{1}}^{t_{2}}\left|\frac{\partial^{2}}{\partial t^{2}} G(\eta, s)\right| h(s) f\left(s, u, u^{\prime}, u^{\prime \prime}\right) d s \\
& \geq M_{4} \min _{t \in[m, M]} u(t) \int_{m}^{M} \frac{h(s) f\left(s, u, u^{\prime}, u^{\prime \prime}\right)}{u} d s \\
& \geq M_{4}\|u\|_{0} \int_{m}^{M} \frac{h(s) f\left(s, u, u^{\prime}, u^{\prime \prime}\right)}{u(s)} d s .
\end{aligned}
$$

The argument guarantees that, for $v \in] 0, \epsilon_{n}[$,

$$
\begin{aligned}
& \frac{\Psi(v)}{v} \\
& =\frac{1}{v} \inf _{u \in \partial \mathscr{K}(v)} \max \left\{\max _{t \in\left[t_{1}, t_{2}\right]}\left|\int_{t_{1}}^{t_{2}} G(t, s) h(s) f\left(s, u, u^{\prime}, u^{\prime \prime}\right) d s\right|,\right.
\end{aligned}
$$

$$
\max _{t \in\left[t_{1}, t_{2}\right]} \mid \int_{t_{1}}^{t_{2}} \frac{\partial}{\partial t} G(t, s)
$$$$
\times h(s) f\left(s, u, u^{\prime}, u^{\prime \prime}\right) d s \mid
$$$$
\max _{t \in\left[t_{1}, t_{2}\right]} \mid \int_{t_{1}}^{t_{2}} \frac{\partial^{2}}{\partial t^{2}} G(t, s)
$$$$
\left.\times h(s) f\left(s, u, u^{\prime}, u^{\prime \prime}\right) d s \mid\right\}
$$

$\geq \frac{1}{v} \inf _{u \in \partial \mathscr{K}(v)} \max \left\{\tau_{1}^{2} M_{4}\|u\|_{0}\right.$

$$
\begin{aligned}
& \times \int_{m}^{M} \frac{h(s) f\left(s, u, u^{\prime}, u^{\prime \prime}\right)}{u} d s, \\
& \tau_{1} \tau_{2} M_{4}\|u\|_{0} \int_{m}^{M} \frac{h(s) f\left(s, u, u^{\prime}, u^{\prime \prime}\right)}{u} d s, \\
& \left.M_{4}\|u\|_{0} \int_{m}^{M} \frac{h(s) f\left(s, u, u^{\prime}, u^{\prime \prime}\right)}{u} d s\right\} \\
& =\max \left\{\frac{1}{v} \inf _{u \in \partial \mathscr{K}(v)} \tau_{1}^{2} M_{4}\|u\|_{0} \int_{m}^{M} \frac{h(s) f\left(s, u, u^{\prime}, u^{\prime \prime}\right)}{u} d s,\right. \\
& \frac{1}{v} \inf _{u \in \partial \mathscr{K}(v)} \tau_{1} \tau_{2} M_{4}\|u\|_{0} \int_{m}^{M} \frac{h(s) f\left(s, u, u^{\prime}, u^{\prime \prime}\right)}{u} d s, \\
& \left.\frac{1}{v} \inf _{u \in \mathscr{K}(v)} M_{4}\|u\|_{0} \int_{m}^{M} \frac{h(s) f\left(s, u, u^{\prime}, u^{\prime \prime}\right)}{u} d s\right\} \\
& \geq \max \left\{\tau_{1}^{2} M_{4} \int_{m}^{M} \frac{h(s) f\left(s, u, u^{\prime}, u^{\prime \prime}\right)}{u} d s,\right. \\
& \tau_{1} \tau_{2} M_{4} \int_{m}^{M} \frac{h(s) f\left(s, u, u^{\prime}, u^{\prime \prime}\right)}{u} d s, \\
& \left.M_{4} \int_{m}^{M} \frac{h(s) f\left(s, u, u^{\prime}, u^{\prime \prime}\right)}{u} d s\right\} \\
& \geq \max \left\{\tau_{1}^{2} M_{4}(M-m) n, \tau_{1} \tau_{2} M_{4}(M-m) n,\right. \\
& \left.M_{4}(M-m) n\right\} \\
& \geq \max \left\{\tau_{1}, \tau_{2}\right\} \tau_{1} M_{4}(M-m) n \text {. }
\end{aligned}
$$

Hence, (60) holds.

Finally, from the definitions of $\Phi$ and $\Psi$, it is easy to check that

$$
\Phi(v)=\sup _{u \in \partial \mathscr{K}(v)}\|T u\|, \quad \Psi(v)=\inf _{u \in \partial \mathscr{K}(v)}\|T u\| .
$$


Note that (56) and (60) imply that there exist $V_{1}, V_{2}$ such that $V_{2} \gg V_{1}$ and $\Phi\left(V_{2}\right) / V_{2} \leq 1, \Psi\left(V_{1}\right) / V_{1} \geq 1$, respectively. Thus, it follows that

$$
\begin{array}{cc}
\|T u\| \geq\|u\|, \quad u \in \partial \mathscr{K}\left(V_{1}\right), \\
\|T u\| \leq\|u\|, \quad u \in \partial \mathscr{K}\left(V_{2}\right) .
\end{array}
$$

By virtue of (66), the hypotheses of Lemma 1 are satisfied; we infer that there exists $u^{*} \in K$ such that $T u^{*}=u^{*}$, and $V_{1} \leq\left\|u^{*}\right\| \leq V_{2}$. In addition, condition $\left(H_{2}\right)$ guarantees that the fixed point is positive, so the problem $\left(\mathscr{P}_{s}\right)$ has at least one positive solution. This completes the proof.

Remark 9. Since the definition of the operator $T$, a slight modification of $M_{4}=\min \left\{\min _{s \in[m, M]} G(\eta, s) h(t)\right.$, $\left.\min _{s \in[m, M]}\left|\left(\partial^{2} / \partial t^{2}\right) G(\eta, s) h(t)\right|\right\}$ and

$\left(C_{2}\right)$ there exist constants $m, M(m<M)$ such that $[m, M] \subseteq[\xi, \omega]$ and satisfy

$$
\liminf _{\mu \rightarrow 0^{+}} \min _{t \in[m, M]} \frac{f(t, \mu, \nu, \omega)}{\mu}=\infty
$$

which guarantee that the problem $\left(\mathscr{P}_{s}\right)$ has at least one positive solution.

Furthermore, $f$ may be superlinear or sublinear; we can give the following existence results for the problem $\left(\mathscr{P}_{s}\right)$ and the proof is omitted.

Theorem 10. Suppose $\left(H_{1}\right)-\left(H_{4}\right)$ hold, $f(t, 0,0,0) \not \equiv 0$ on any subinterval of $\left[t_{1}, t_{2}\right]$ and

$$
\begin{aligned}
& \left(D_{1}\right) \underline{f}_{0}=0 \text { and } \bar{f}_{\infty}=\infty \text {, or } \\
& \left(D_{2}\right) \underline{f}_{\infty}=0 \text { and } \bar{f}_{0}=\infty .
\end{aligned}
$$

Then, the problem $\left(\mathscr{P}_{s}\right)$ has at least one positive solution.

\section{Multiple Solutions}

Let the nonnegative continuous convex functionals $\gamma$ and $\theta$, the nonnegative continuous concave functional $\alpha$, and the nonnegative continuous functional $\psi$ be defined on the cone $K$ by

$$
\begin{gathered}
\gamma(u)=\max _{t \in\left[t_{1}, t_{2}\right]}\left|u^{\prime \prime}(t)\right|, \\
\theta(u)=\max _{t \in\left[t_{1}, t_{2}\right]}|u(t)|, \\
\alpha(u)=\min _{t \in[\xi, \omega]} u(t), \\
\psi(u)=\max _{t \in\left[t_{1}, t_{2}\right]}\left|u^{\prime}(t)\right| .
\end{gathered}
$$

To prove our main results, we recommend notation

$$
\begin{aligned}
N_{1}= & \max \left\{\int_{t_{1}}^{\eta}-\frac{\partial^{2}}{\partial t^{2}} x_{1}(\eta, s) h(s) d s,\right. \\
& \left.\int_{t_{1}}^{\eta} \frac{\partial^{2}}{\partial t^{2}} y_{1}\left(t_{1}, s\right) h(s) d s\right\} \\
+ & \max \left\{\int_{\eta}^{t_{2}}-\frac{\partial^{2}}{\partial t^{2}} x_{2}\left(t_{2}, s\right) h(s) d s,\right. \\
N_{2}= & \tau_{1}\left(\max \left\{\int_{t_{1}}^{\eta} x_{1}(\eta, s) h(s) d s, \int_{t_{1}}^{\eta} y_{1}\left(t_{1}, s\right) h(s) d s\right\}\right. \\
& +\max _{2}\left\{\int_{\eta}^{t_{2}} x_{2}(\eta, s) h(s) d s,\right. \\
+ & \max \left\{\int_{\eta}^{t_{2}}-\frac{\partial}{\partial t} x_{2}\left(t_{2}, s\right) h(s) d s,\right. \\
N_{3}= & \left.\left.\max \left\{\int_{t_{1}}^{\eta} \frac{\partial}{\partial t} x_{1}\left(t_{1}, s\right) h(s) d s, \int_{t_{1}}^{\eta}-\frac{\partial}{\partial t} y_{2}\left(t_{2}, s\right) h(s) d s\right\} . s\right) h(s) d s\right\}
\end{aligned}
$$

Now, we are ready to apply Avery and Peterson fixed point theorem to the operator $T$ to give the sufficient conditions for the existence of at least three positive solutions to the problem $\left(\mathscr{P}_{s}\right)$.

Theorem 11. Let $\left(H_{1}\right)-\left(H_{4}\right)$ hold; $f(t, 0,0,0)$ p 0 on any subinterval of $\left[t_{1}, t_{2}\right]$. Suppose that there exist numbers $m_{1}, m_{2}$, $m_{4}$ such that $0<m_{1}<m_{2} \leq \tau_{1}^{2} \vartheta / \max \left\{\left|6 t_{1}-\left(2 \lambda_{2} / \lambda_{1}\right)\right|, \mid 6 t_{2}-\right.$ $\left.\left(2 \lambda_{2} / \lambda_{1}\right) \mid\right\} m_{4}, \min \left\{-\xi^{3}+\left(\lambda_{2} / \lambda_{1}\right) \xi^{2}+\left(\lambda_{3} / \lambda_{1}\right) \xi+\lambda_{4},-\omega^{3}+\right.$ $\left.\left(\lambda_{2} / \lambda_{1}\right) \omega^{2}+\left(\lambda_{3} / \lambda_{1}\right) \omega+\lambda_{4},-\eta^{3}+\left(\lambda_{2} / \lambda_{1}\right) \eta^{2}+\left(\lambda_{3} / \lambda_{1}\right) \eta+\lambda_{4}\right\} \geq$ $\tau_{1} \vartheta$, and

$\left(E_{1}\right) f(t, \mu, \nu, \omega) \leq m_{4} / N_{1}$ for $(t, \mu, v, \omega) \in\left[t_{1}, t_{2}\right] \times$ $\left[0, \tau_{3} \tau_{4} m_{4}\right] \times\left[-\tau_{4} m_{4}, \tau_{4} m_{4}\right] \times\left[-m_{4}, m_{4}\right]$,

$\left(E_{2}\right) f(t, \mu, \nu, \omega)>m_{2} / N_{2}$ for $(t, \mu, \nu, \omega) \in[\xi, \omega] \times$ $\left[m_{2}, m_{2} \tau_{1}^{-2}\right] \times\left[-\tau_{4} m_{4}, \tau_{4} m_{4}\right] \times\left[-m_{4}, m_{4}\right]$,

$\left(E_{3}\right) f(t, \mu, \nu, \omega) \leq m_{1} / N_{3}$ for $(t, \mu, v, \omega) \in\left[t_{1}, t_{2}\right] \times$ $\left[0, \tau_{3} \tau_{4} m_{4}\right] \times\left[-\tau_{4} m_{4}, m_{1}\right] \times\left[-m_{4}, m_{4}\right]$. 
Then, the $\left(\mathscr{P}_{s}\right)$ has at least three positive solutions $u_{1}, u_{2}, u_{3} \in$ $K$ such that

$$
\begin{gathered}
\max _{t \in\left[t_{1}, t_{2}\right]}\left|u_{i}^{\prime \prime}(t)\right| \leq m_{4} \quad \text { for } i=1,2,3, \\
m_{2}<\min _{t \in[\xi, \omega]} u_{1}(t), \\
m_{1}<\max _{t \in\left[t_{1}, t_{2}\right]} u_{2}^{\prime}(t) \quad \text { with } \min _{t \in[\xi, \omega]} u_{2}(t)<m_{2}, \\
\max _{t \in\left[t_{1}, t_{2}\right]} u_{3}^{\prime}(t)<m_{1} .
\end{gathered}
$$

Proof. By the definition of operator $T$ and its properties, it suffices to show that the conditions of Lemma 2 hold with respect to $T$. Furthermore, from the fact that $G(t, s)$ is the Green function, it is not difficult to prove that a fixed point of $T$ is coincident with the solution of the boundary value problem $\left(\mathscr{P}_{s}\right)$, so we concentrate on the existence of the fixed point of the operator $T$.

The functionals are defined as (68); we also note that $\alpha, \gamma, \theta$, and $\psi$ are continuous nonnegative functionals on $K$ satisfying $\psi(\lambda u) \leq \lambda \psi(u)$ for $0 \leq \lambda \leq 1$ such that, for some positive numbers $\epsilon$ and $m_{4}, \alpha(u) \leq \theta(u)=\psi(u)$ and $\|u\|_{0} \leq \epsilon \gamma(u)$, for $u \in \overline{P\left(\gamma, m_{4}\right)}$, where $\epsilon \leq 1$. If $u \in \overline{P\left(\gamma, m_{4}\right)}$, then $\gamma(u)=\max _{t \in\left[t_{1}, t_{2}\right]}\left|u^{\prime \prime}(t)\right| \leq m_{4}$. We get $\max _{t \in\left[t_{1}, t_{2}\right]}|u(t)| \leq \tau_{3} \max _{t \in\left[t_{1}, t_{2}\right]}\left|u^{\prime}(t)\right| \leq \tau_{3} \tau_{4} m_{4}$ by (39). It follows from assumption $\left(E_{1}\right)$ that $f\left(t, u(t), u^{\prime}(t), u^{\prime \prime}(t)\right) \leq$ $m_{4} / N_{1}$. On the other hand, for $u \in K$, we have proved that $T u \in K$ in Lemma 7 and thus

$$
\begin{aligned}
& \gamma(T u) \\
& =\max _{t \in\left[t_{1}, t_{2}\right]}\left|(T u)^{\prime \prime}(t)\right| \\
& \leq \max _{t \in\left[t_{1}, t_{2}\right]}\left|\int_{t_{1}}^{\eta} \frac{\partial^{2}}{\partial t^{2}} G(t, s) h(s) f\left(s, u, u^{\prime}, u^{\prime \prime}\right) d s\right| \\
& +\max _{t \in\left[t_{1}, t_{2}\right]}\left|\int_{\eta}^{t_{2}} \frac{\partial^{2}}{\partial t^{2}} G(t, s) h(s) f\left(s, u, u^{\prime}, u^{\prime \prime}\right) d s\right| \\
& =\max _{2}\left\{\int_{t_{1}}^{\eta}-\frac{\partial^{2}}{\partial t^{2}} x_{1}(\eta, s) h(s) f\left(s, u, u^{\prime}, u^{\prime \prime}\right) d s,\right. \\
& \left.\quad \int_{t_{1}}^{\eta} \frac{\partial^{2}}{\partial t^{2}} y_{1}\left(t_{1}, s\right) h(s) f\left(s, u, u^{\prime}, u^{\prime \prime}\right) d s\right\} \\
& +\max \left\{\int_{\eta}^{t_{2}}-\frac{\partial^{2}}{\partial t^{2}} x_{2}\left(t_{2}, s\right) h(s) f\left(s, u, u^{\prime}, u^{\prime \prime}\right) d s,\right. \\
& \left.\int_{\eta}^{t_{2}} \frac{\partial^{2}}{\partial t^{2}} y_{2}(\eta, s) h(s) f\left(s, u, u^{\prime}, u^{\prime \prime}\right) d s\right\}
\end{aligned}
$$

$$
\begin{gathered}
\leq \frac{m_{4}}{N_{1}}\left(\operatorname { m a x } \left\{\int_{t_{1}}^{\eta}-\frac{\partial^{2}}{\partial t^{2}} x_{1}(\eta, s) h(s) d s,\right.\right. \\
\left.\int_{t_{1}}^{\eta} \frac{\partial^{2}}{\partial t^{2}} y_{1}\left(t_{1}, s\right) h(s) d s\right\} \\
+\max \left\{\int_{\eta}^{t_{2}}-\frac{\partial^{2}}{\partial t^{2}} x_{2}\left(t_{2}, s\right) h(s) d s,\right. \\
\left.\left.\int_{\eta}^{t_{2}} \frac{\partial^{2}}{\partial t^{2}} y_{2}(\eta, s) h(s) d s\right\}\right) \\
=m_{4} .
\end{gathered}
$$

Therefore, $T: \overline{P\left(\gamma, m_{4}\right)} \rightarrow \overline{P\left(\gamma, m_{4}\right)}$.

Firstly, to verify the condition $\left(B_{1}\right)$ of Lemma 2 holds, we choose $u_{0}$ which is defined by (46); (47) gives the expression of $\vartheta$. Set $\bar{m}=m_{2} /\left(\tau_{1}^{2} \vartheta\right)$; we have

$$
\begin{aligned}
& \alpha\left(u_{0}\right)=\min _{t \in[\xi, \omega]}\left|u_{0}(t)\right| \\
&=\bar{m} \min \left\{-\xi^{3}+\frac{\lambda_{2}}{\lambda_{1}} \xi^{2}+\frac{\lambda_{3}}{\lambda_{1}} \xi+\lambda_{4},\right. \\
&-\omega^{3}+\frac{\lambda_{2}}{\lambda_{1}} \omega^{2}+\frac{\lambda_{3}}{\lambda_{1}} \omega+\lambda_{4}, \\
&\left.-\eta^{3}+\frac{\lambda_{2}}{\lambda_{1}} \eta^{2}+\frac{\lambda_{3}}{\lambda_{1}} \eta+\lambda_{4}\right\}
\end{aligned}
$$

$$
\begin{aligned}
& \geq \tau_{1} \bar{m} \vartheta=\frac{m_{2}}{\tau_{1}}>m_{2}, \\
\theta\left(u_{0}\right) & =\max _{t \in\left[t_{1}, t_{2}\right]}\left|u_{0}(t)\right|=\bar{m} \vartheta=\frac{m_{2}}{\tau_{1}^{2}}>m_{2}, \\
\gamma\left(u_{0}\right) & =\max _{t \in\left[t_{1}, t_{2}\right]}\left|u_{0}^{\prime \prime}(t)\right| \\
& =\bar{m} \max \left\{\left|6 t_{1}-\frac{2 \lambda_{2}}{\lambda_{1}}\right|,\left|6 t_{2}-\frac{2 \lambda_{2}}{\lambda_{1}}\right|\right\} \\
& =\frac{\max \left\{\left|6 t_{1}-\left(2 \lambda_{2} / \lambda_{1}\right)\right|,\left|6 t_{2}-\left(2 \lambda_{2} / \lambda_{1}\right)\right|\right\}}{\vartheta} \frac{m_{2}}{\tau_{1}^{2}} \\
& \leq m_{4} .
\end{aligned}
$$

Equation (72) implies that $\alpha\left(u_{0}\right) \geq \tau_{1} \theta\left(u_{0}\right)$; that is, $\min _{t \in[\xi, \omega]} u_{0}(t) \geq \tau_{1} \max _{t \in\left[t_{1}, t_{2}\right]}\left|u_{0}(t)\right|$. So $u_{0} \in K$; it holds $u_{0} \in P\left(\gamma, \theta, \alpha, m_{2}, m_{2} \tau_{1}^{-2}, m_{4}\right)$ and $\left\{P\left(\gamma, \theta, \alpha, m_{2}, m_{2} \tau_{1}^{-2}, m_{4}\right) \mid\right.$ $\left.\alpha\left(u_{0}\right)>m_{2}\right\} \neq \emptyset$. Hence, if $u \in P\left(\gamma, \theta, \alpha, m_{2}, m_{2} \tau_{1}^{-2}, m_{4}\right)$, then 
$m_{2} \leq u(t) \leq m_{2} \tau_{1}^{-2},\left|u^{\prime \prime}(t)\right| \leq m_{4}$ for $t \in[\xi, \omega]$. In view of assumption $\left(E_{2}\right)$, we get

$$
\begin{aligned}
& \alpha(T u) \\
& =\min _{t \in[\xi, \omega]}(T u)(t) \\
& =\min \left\{\int_{t_{1}}^{\eta} x_{1}(\xi, s) h(s) f\left(s, u, u^{\prime}, u^{\prime \prime}\right) d s,\right. \\
& \int_{t_{1}}^{\eta} y_{1}(\omega, s) h(s) f\left(s, u, u^{\prime}, u^{\prime \prime}\right) d s, \\
& \int_{\eta}^{t_{2}} x_{2}(\omega, s) h(s) f\left(s, u, u^{\prime}, u^{\prime \prime}\right) d s, \\
& \int_{\eta}^{t_{2}} y_{2}(\xi, s) h(s) f\left(s, u, u^{\prime}, u^{\prime \prime}\right) d s, \\
& \int_{t_{1}}^{\eta} y_{1}(\eta, s) h(s) f\left(s, u, u^{\prime}, u^{\prime \prime}\right) d s, \\
& \left.\int_{\eta}^{t_{2}} y_{2}(\eta, s) h(s) f\left(s, u, u^{\prime}, u^{\prime \prime}\right) d s\right\} \\
& \geq \tau_{1}\left(\operatorname { m a x } \left\{\int_{t_{1}}^{\eta} x_{1}(\eta, s) h(s) f\left(s, u, u^{\prime}, u^{\prime \prime}\right) d s,\right.\right. \\
& \left.\int_{t_{1}}^{\eta} y_{1}\left(t_{1}, s\right) h(s) f\left(s, u, u^{\prime}, u^{\prime \prime}\right) d s\right\} \\
& +\max \left\{\int_{\eta}^{t_{2}} x_{2}(\eta, s) h(s) f\left(s, u, u^{\prime}, u^{\prime \prime}\right) d s\right. \\
& \left.\left.\int_{\eta}^{t_{2}} y_{2}\left(t_{2}, s\right) h(s) f\left(s, u, u^{\prime}, u^{\prime \prime}\right) d s\right\}\right) \\
& \geq \frac{m_{2}}{N_{2}} \tau_{1}\left(\operatorname { m a x } \left\{\int_{t_{1}}^{\eta} x_{1}(\eta, s) h(s) d s,\right.\right. \\
& \left.\int_{t_{1}}^{\eta} y_{1}\left(t_{1}, s\right) h(s) d s\right\} \\
& +\max \left\{\int_{\eta}^{t_{2}} x_{2}(\eta, s) h(s) d s,\right. \\
& \left.\left.\int_{\eta}^{t_{2}} y_{2}\left(t_{2}, s\right) h(s) d s\right\}\right) \\
& =m_{2} \text {. }
\end{aligned}
$$

Thus, $\alpha(T u)>m_{2}$ for each $u \in P\left(\gamma, \theta, \alpha, m_{2}, m_{2} \tau_{1}^{-2}, m_{4}\right)$.

Next, we verify that the condition $\left(B_{2}\right)$ of Lemma 2 is satisfied. In fact, if $u \in P\left(\gamma, \alpha, m_{2}, m_{4}\right)$ with $\theta(T u)>m_{2} \tau_{1}^{-2}$, then

$$
\begin{aligned}
\alpha(T u) & =\min _{t \in[\xi, \omega]}(T u)(t) \geq \tau_{1} \max _{t \in\left[t_{1}, t_{2}\right]}(T u)(t) \\
& =\tau_{1} \theta(T u)>m_{2} .
\end{aligned}
$$

So $\alpha(T u)>m_{2}$ for any $u \in P\left(\gamma, \alpha, m_{2}, m_{4}\right)$ with $\vartheta(T u)>$ $m_{2} \tau_{1}^{-2}$.

Finally, we assert that the condition $\left(B_{3}\right)$ of Lemma 2 is fulfilled. It is clear that $0 \notin Q\left(\gamma, \psi, m_{1}, m_{4}\right)$. Suppose that $u \in$ $Q\left(\gamma, \psi, m_{1}, m_{4}\right)$ with $\psi(u)=m_{1}$; by assumption $\left(E_{3}\right)$, we have

$$
\begin{aligned}
& \psi(T u) \\
& =\max _{t \in\left[t_{1}, t_{2}\right]}\left|(T u)^{\prime}(t)\right| \\
& \leq \max _{t \in\left[t_{1}, t_{2}\right]} \int_{t_{1}}^{\eta}\left|\frac{\partial}{\partial t} G(t, s)\right| h(s) f\left(s, u, u^{\prime}, u^{\prime \prime}\right) d s \\
& +\max _{t \in\left[t_{1}, t_{2}\right]} \int_{\eta}^{t_{2}}\left|\frac{\partial}{\partial t} G(t, s)\right| h(s) f\left(s, u, u^{\prime}, u^{\prime \prime}\right) d s \\
& =\max \left\{\int_{t_{1}}^{\eta} \frac{\partial}{\partial t} x_{1}\left(t_{1}, s\right) h(s) f\left(s, u, u^{\prime}, u^{\prime \prime}\right) d s,\right. \\
& \left.\int_{t_{1}}^{\eta}-\frac{\partial}{\partial t} y_{1}\left(t_{1}, s\right) h(s) f\left(s, u, u^{\prime}, u^{\prime \prime}\right) d s\right\} \\
& +\max \left\{\int_{\eta}^{t_{2}}-\frac{\partial}{\partial t} x_{2}\left(t_{2}, s\right) h(s) f\left(s, u, u^{\prime}, u^{\prime \prime}\right) d s,\right. \\
& \left.\int_{\eta}^{t_{2}} \frac{\partial}{\partial t} y_{2}\left(t_{2}, s\right) h(s) f\left(s, u, u^{\prime}, u^{\prime \prime}\right) d s\right\} \\
& \leq \frac{m_{1}}{N_{3}}\left(\operatorname { m a x } \left\{\int_{t_{1}}^{\eta} \frac{\partial}{\partial t} x_{1}\left(t_{1}, s\right) h(s) d s,\right.\right. \\
& \left.\int_{t_{1}}^{\eta}-\frac{\partial}{\partial t} y_{1}\left(t_{1}, s\right) h(s) d s\right\} \\
& +\max \left\{\int_{\eta}^{t_{2}}-\frac{\partial}{\partial t} x_{2}\left(t_{2}, s\right) h(s) d s,\right. \\
& \left.\left.\int_{\eta}^{t_{2}} \frac{\partial}{\partial t} y_{2}\left(t_{2}, s\right) h(s) d s\right\}\right) \\
& =m_{1} \text {. }
\end{aligned}
$$

Hence, $\psi(T u) \leq m_{1}$ for $u \in Q\left(\gamma, \psi, m_{1}, m_{4}\right)$ with $\psi(u)=m_{1}$.

Hence, the hypotheses of Lemma 2 are satisfied. Therefore, we can conclude that the operator $T$ has three positive fixed points $u_{1}, u_{2}, u_{3} \in \overline{P\left(\gamma, m_{4}\right)}$. In addition, condition $\left(A_{2}\right)$ guarantees that those fixed points are positive, so the problem $\left(\mathscr{P}_{s}\right)$ has at least three positive solutions $u_{1}(t), u_{2}(t)$, $u_{3}(t)$ satisfying $\max _{t \in\left[t_{1}, t_{2}\right]}\left|u_{i}^{\prime \prime}(t)\right| \leq m_{4}$, for $i=1,2,3$, $m_{2}<\min _{t \in[\xi, \omega]} u_{1}(t)$, and $m_{1}<\max _{t \in\left[t_{1}, t_{2}\right]} u_{2}^{\prime}(t)$ with $\min _{t \in[\xi, \omega]} u_{2}(t)<m_{2}, \max _{t \in\left[t_{1}, t_{2}\right]} u_{3}^{\prime}(t)<m_{1}$. 


\section{Applications}

The known Falkner-Skan equation

$$
u^{\prime \prime \prime}(t)+\frac{1}{2}(\varrho+1) u^{\prime \prime}(t) u(t)+\rho\left(1-u^{\prime}(t)^{2}\right)=0
$$

whose solution is the similarity solution of the two-dimensional incompressible laminar boundary-layer equations [4, 5]. When $\varrho=1$, which arises in the study of two-dimensional incompressible viscous flow past a thin semi-infinite flat plate $\rho \pi, 0 \leq \rho \leq 1$. The special case $\rho=0$ is Blasius's equation, in which the wedge reduces to a flat plate. The special case $\rho=1 / 2$ is called Homann's equation, in which the wedge reduces to a flat plate. The special case $\rho=1$ is called Hiemenz's equation. $\rho>0$ corresponds to flow toward the wedge, otherwise, to flow away from the wedge. When $\varrho \neq 1$, taking $\varrho=\rho$, Blasius flow over a flat plate with a sharp edge as $\rho=0$, flow over a wedge with half angle $\theta_{1 / 2}=\rho \pi /(\rho+1)$, $0<\theta_{1 / 2}<\pi / 2$ as $0<\rho<1$, Hiemenz flow toward a plane stagnation point as $\rho=1$, flow into a corner with $\theta_{1 / 2}>\pi / 2$ as $1<\rho<2$, and no corresponding simple ideal flow as $\rho>2$ exist.

Now, we give a general equation of (77), subject to the boundary conditions. But it is even more difficult to solve, because it is singular; the solution $u(t)$ denotes the physically relevant solutions for $\varrho=1$ and the dimensionless stream function for $\varrho \neq 1$ of (77), respectively.

Consider

$$
\begin{array}{r}
u^{\prime \prime \prime}(t)+h(t) f\left(t, u(t), u^{\prime}(t), u^{\prime \prime}(t)\right)=0, \\
0<t<1, \\
u(0)-u^{\prime \prime}(0)=u^{\prime}\left(\frac{1}{4}\right)=u(1)+u^{\prime}(1)=0,
\end{array}
$$

where

$$
h(t)=\frac{1}{\sqrt{t(1-t)}},
$$

$$
\begin{aligned}
& f(t, \mu, \nu, \omega) \\
& = \begin{cases}\sqrt{1+t^{2}}\left(\mu^{2 / 3}+\frac{2}{\pi} \arctan \left(1+v^{2} \omega^{4}\right)\right), & 0 \leq \mu \leq 1, \\
\sqrt{1+t^{2}}\left(1+\frac{2}{\pi} \arctan \left(1+\nu^{2} \omega^{4}\right)\right), & \mu>1 .\end{cases}
\end{aligned}
$$

It is easy to check that hypotheses $\left(C_{1}\right),\left(C_{2}\right)$ hold. Taking $L=1,[m, M]=[1 / 3,2 / 5] \subseteq[\xi, \omega]=[1 / 4,1 / 2], F(t)=1$, by some calculations, we have $M_{1}=(33 / 16) \sqrt{2 \pi}, M_{2}=1$, $M_{3}=1, M_{4}=17 / 36, \tau_{1}=8 / 11$, and

$$
\begin{aligned}
& G(t, s) \\
& s \in\left[0, \frac{1}{4}\right]\left\{\begin{array}{l}
\frac{1}{16}\left(2 t^{2}-t+4\right)\left(s^{2}+2\right) \\
+t\left(\frac{1}{4}-s\right)-\frac{1}{2}(t-s)^{2}, \quad s \leq t, \\
\frac{1}{16}\left(2 t^{2}-t+4\right)\left(s^{2}+2\right)
\end{array}\right. \\
& s \in\left[\frac{1}{4}, 1\right]\left\{\begin{array}{l}
+t\left(\frac{1}{4}-s\right), \quad t \leq s, \\
\frac{1}{16}\left(2 t^{2}-t+4\right)\left(s^{2}-4 s+3\right) \\
\frac{1}{16}\left(2 t^{2}-t+4\right)\left(s^{2}-4 s+3\right),
\end{array}\right. \\
& \quad\left\{\begin{array}{l}
\frac{1}{16}(t, \\
2
\end{array}\right.
\end{aligned}
$$

then $f^{2}(t, \mu, v, \omega) \leq 1$ for $(t, \mu, v, \omega) \in[0,1] \times[1, \infty[\times]-\infty$, $\infty\left[^{2}\right.$ and $\lim \inf _{\mu \rightarrow 0^{+}} \min _{t \in[1 / 4,1 / 2]}(h f(t, \mu, \nu, \omega) / \mu)=\infty$. By Theorem 8 , problem (78) has at least one positive solution. These results accord with $\varrho=1$; the solutions of (77) are known to exist [23].

On the other hand, let

$$
\begin{aligned}
& f(t, \mu, \nu, \omega) \\
& = \begin{cases}10^{-2} t+\frac{3}{4} \mu^{2}+\left(\frac{\nu \omega}{10^{10}}\right)^{3}, & 0<\mu<3.78, \\
10^{-2} t+\frac{3}{4} \times 3.78^{2}+\left(\frac{\nu \omega}{10^{10}}\right)^{3}, & \mu \geq 3.78 .\end{cases}
\end{aligned}
$$

It is easy to check that hypotheses $\left(E_{1}\right)-\left(E_{3}\right)$ hold and $f(t, 0,0,0) \not \equiv 0$ on $[0,1]$. By some calculations, we have $k=$ $4, \tau_{1}=8 / 11, \tau_{3}=8.992, \tau_{4}=3 / 4, \lambda_{1}=4, \lambda_{2}=29 / 8, \lambda_{3}=$ $-15 / 16, \lambda_{4}=29 / 16, \vartheta=29 / 16, \min \{1.795,1.797,1.795\} \geq$ $\tau_{1} \vartheta \approx 1.318, N_{1} \approx 2.238, N_{2} \approx 0.730, N_{3} \approx 0.505$. If we choose $m_{1}=1, m_{2}=2, m_{4}=10^{5}$, then $f(t, \mu, v, \omega)$ satisfies

$$
\begin{aligned}
f(t, \mu, \nu, \omega) \leq & \frac{m_{4}}{N_{1}} \approx 4.468 \times 10^{4}, \\
(t, \mu, \nu, \omega) \in[0,1] \times[0,674400] & \\
& \times\left[-7.5 \times 10^{4}, 7.5 \times 10^{4}\right] \times\left[-10^{5}, 10^{5}\right], \\
f(t, \mu, v, \omega)> & \frac{m_{2}}{N_{2}} \approx 2.740,
\end{aligned}
$$




$$
\begin{aligned}
&(t, \mu, \nu, \omega) \in\left[\frac{1}{4}, \frac{1}{2}\right] \times[2,3.78] \\
& \times\left[-7.5 \times 10^{4}, 7.5 \times 10^{4}\right] \times\left[-10^{5}, 10^{5}\right] \\
& f(t, \mu, v, \omega) \leq \frac{m_{1}}{N_{3}} \approx 1.980 \\
&(t, \mu, \nu, \omega) \in[0,1] \times[0,1] \\
& \quad \times\left[-7.5 \times 10^{4}, 1\right] \times\left[-10^{5}, 10^{5}\right] .
\end{aligned}
$$

Then all hypotheses of Theorem 11 hold. Hence, problem (78) has at least three positive solutions $u_{1}(t), u_{2}(t)$, and $u_{3}(t)$ such that $\max _{0 \leq t \leq 1}\left|u_{i}^{\prime \prime}(t)\right| \leq 10^{4}(i=1,2,3), 1<\min _{1 / 4 \leq t \leq 3 / 4} u_{1}(t)$, $1 / 2<\max _{0 \leq t \leq 1} u_{2}^{\prime}(t)$ with $\min _{1 / 4 \leq t \leq 3 / 4} u_{2}(t)<1$, $\max _{0 \leq t \leq 1} u_{3}^{\prime}(t)<1 / 2$ hold. These results coincide with $\varrho \neq 1$; the solutions of (77) are the dimensionless stream functions.

\section{Conflict of Interests}

The author declares that there is no conflict of interests.

\section{Authors' Contribution}

The author plotted this paper carefully, gave a rigorous derivation process, read, and approved the final paper.

\section{References}

[1] J. W. Bebernes and D. R. Kassoy, "A mathematical analysis of blowup for thermal reactions-the spatially nonhomogeneous case," SIAM Journal on Applied Mathematics, vol. 40, no. 3, pp. 476-484, 1981.

[2] A. Nachman and A. Callegari, "A nonlinear singular boundary value problem in the theory of pseudoplastic fluids," SIAM Journal on Applied Mathematics, vol. 38, no. 2, pp. 275-281, 1980.

[3] S. H. Lin, "Oxygen diffusion in a spherical cell with nonlinear oxygen uptake kinetics," Journal of Theoretical Biology, vol. 60, no. 2, pp. 449-457, 1976.

[4] M. Moghimi, H. Khoramishad, H. R. Massah, and S. M. Mortezaei, "Approximate analytical solution to flow over a flat plate by variational iteration method," Mechanical and Aerospace Engineering Journal, vol. 2, pp. 63-69, 2006.

[5] H. Nasr, I. A. Hassanien, and H. M. El-Hawary, "Chebyshev solution of laminar boundary layer flow," International Journal of Computer Mathematics, vol. 33, pp. 127-132, 1990.

[6] R. Naz, F. M. Mahomed, and D. P. Mason, "Symmetry solutions of a third-order ordinary differential equation which arises from Prandtl boundary layer equations," Journal of Nonlinear Mathematical Physics, vol. 15, pp. 179-191, 2008.

[7] R. P. Agarwal and D. O'Regan, Singular Differential and Integral Equations with Applications, Kluwer Academic Publishers, Dordrecht, The Netherlands, 2003.

[8] A. Cabada and S. Lois, "Existence of solution for discontinuous third order boundary value problems," Journal of Computational and Applied Mathematics, vol. 110, no. 1, pp. 105-114, 1999.

[9] J. R. Graef and B. Yang, "Multiple positive solutions to a three point third order boundary value problem," Discrete and Continuous Dynamical Systems, vol. 2005, pp. 337-344, 2005.
[10] L. K. Jackson, "Existence and uniqueness of solutions of boundary value problems for third order differential equations," Journal of Differential Equations, vol. 13, pp. 432-437, 1973.

[11] D. R. Anderson and J. M. Davis, "Multiple solutions and eigenvalues for third-order right focal boundary value problems," Journal of Mathematical Analysis and Applications, vol. 267, no. 1, pp. 135-157, 2002.

[12] Y. Guo and W. Ge, "Positive solutions for three-point boundary value problems with dependence on the first order derivative," Journal of Mathematical Analysis and Applications, vol. 290, no. 1, pp. 291-301, 2004.

[13] Z. Liu, L. Debnath, and S. M. Kang, "Existence of monotone positive solutions to a third order two-point generalized right focal boundary value problem," Computers \& Mathematics with Applications, vol. 55, no. 3, pp. 356-367, 2008.

[14] D. R. Anderson, "Green's function for a third-order generalized right focal problem," Journal of Mathematical Analysis and Applications, vol. 288, no. 1, pp. 1-14, 2003.

[15] F. M. Minhós, "On some third order nonlinear boundary value problems: existence, location and multiplicity results," Journal of Mathematical Analysis and Applications, vol. 339, no. 2, pp. 1342-1353, 2008.

[16] R. P. Agarwal, Focal Boundary Value Problems for Differential and Difference Equations, vol. 436 of Mathematics and Its Applications, Kluwer Academic Publishers, Dordrecht, The Netherlands, 1998.

[17] R. P. Agarwal, D. O’Regan, and V. Lakshmikantham, "Singular $(p, n-p)$ focal and $(n, p)$ higher order boundary value problems," Nonlinear Analysis: Theory, Methods \& Applications, vol. 42, no. 2, pp. 215-228, 2000.

[18] A. Cabada and S. Heikkilä, "Extremality and comparison results for discontinuous third order functional initial-boundary value problems," Journal of Mathematical Analysis and Applications, vol. 255, no. 1, pp. 195-212, 2001.

[19] P. J. Y. Wong and R. P. Agarwal, "Multiple positive solutions of two-point right focal boundary value problems," Mathematical and Computer Modelling, vol. 28, no. 3, pp. 41-49, 1998.

[20] D. J. Guo and V. Lakshmikantham, Nonlinear Problems in Abstract Cones, vol. 5 of Notes and Reports in Mathematics in Science and Engineering, Academic Press, Boston, Mass, USA, 1988.

[21] R. W. Leggett and L. R. Williams, "Multiple positive fixed points of nonlinear operators on ordered Banach spaces," Indiana University Mathematics Journal, vol. 28, no. 4, pp. 673-688, 1979.

[22] R. I. Avery and A. C. Peterson, "Three positive fixed points of nonlinear operators on ordered Banach spaces," Computers \& Mathematics with Applications, vol. 42, no. 3-5, pp. 313-322, 2001.

[23] R. L. Panton, Incompressible Flow, John Wiley \& Sons, New York, NY, USA, 1996. 


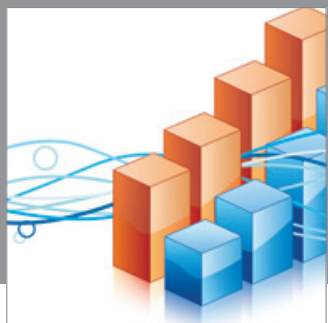

Advances in

Operations Research

mansans

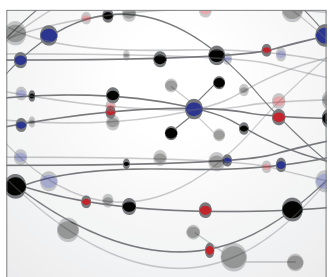

The Scientific World Journal
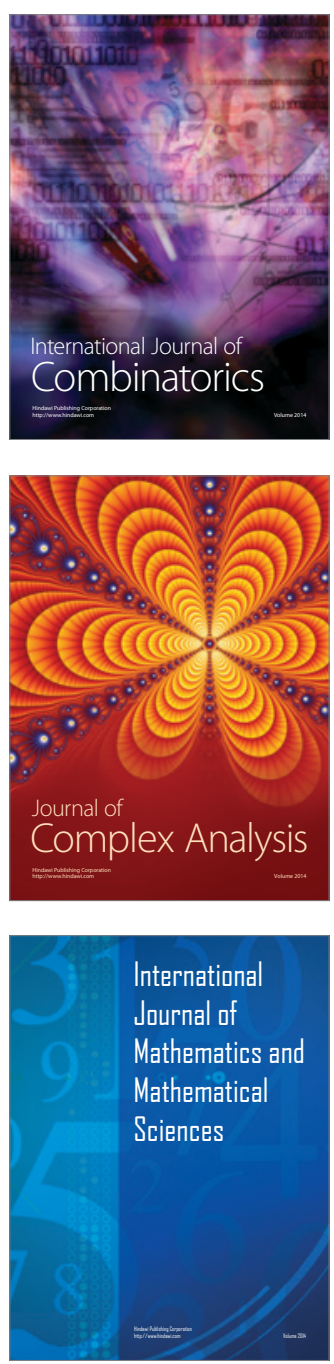
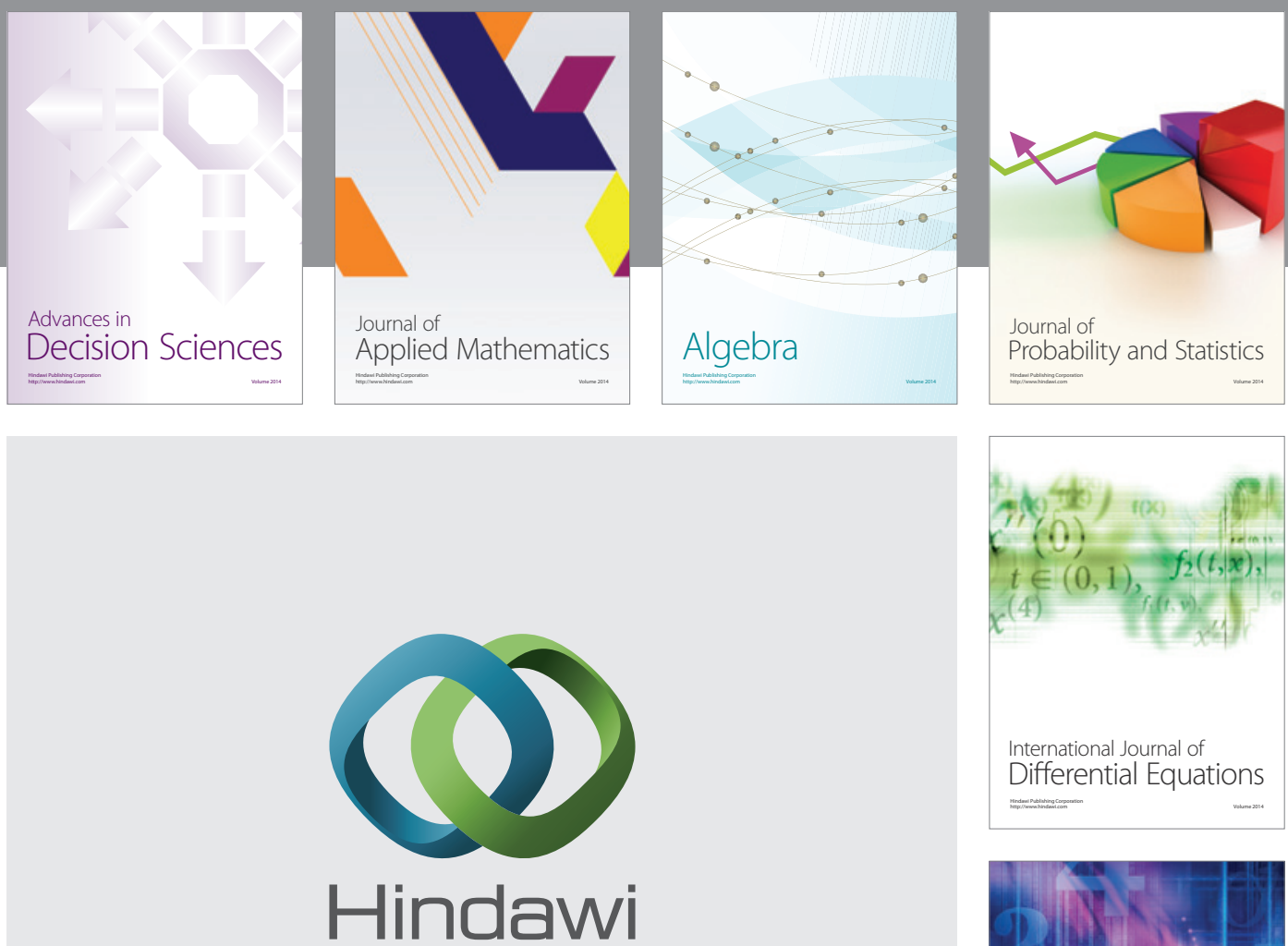

Submit your manuscripts at http://www.hindawi.com
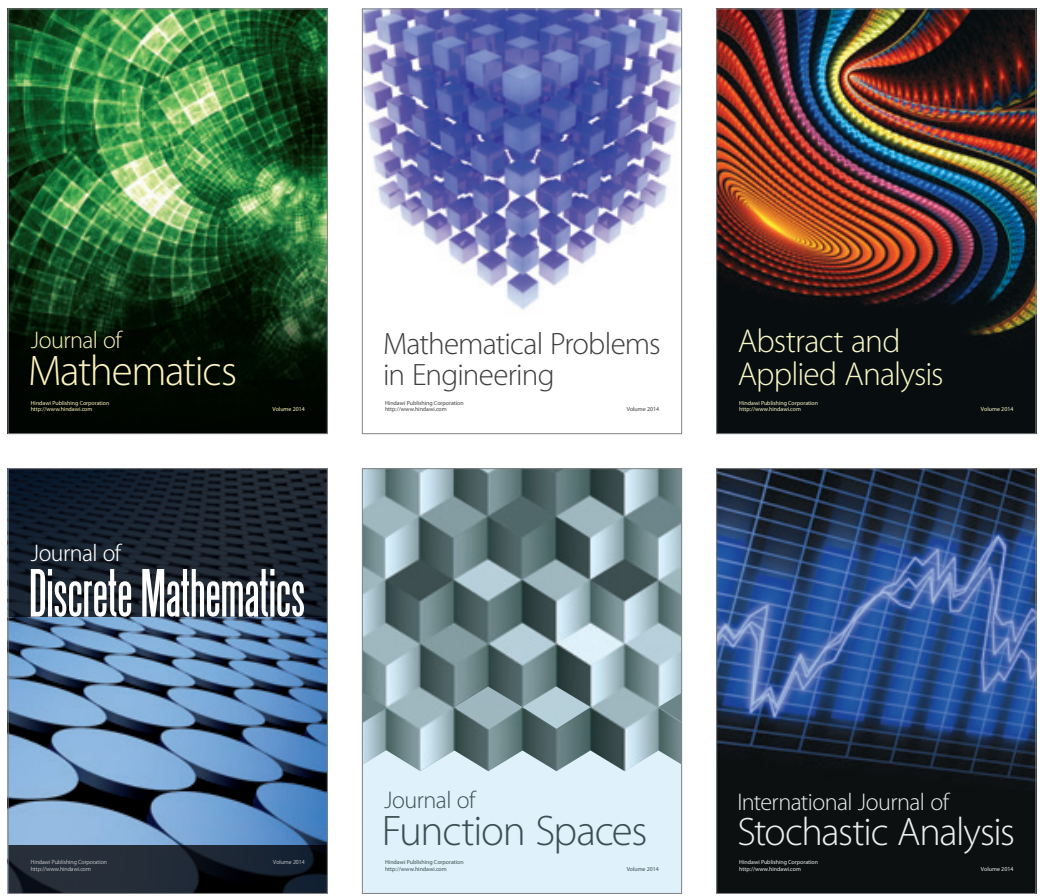

Journal of

Function Spaces

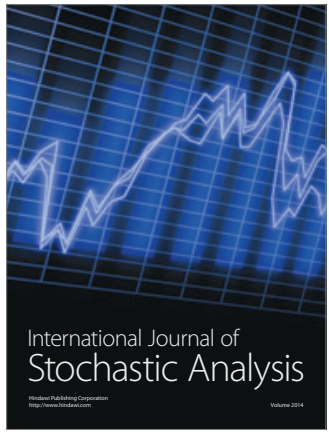

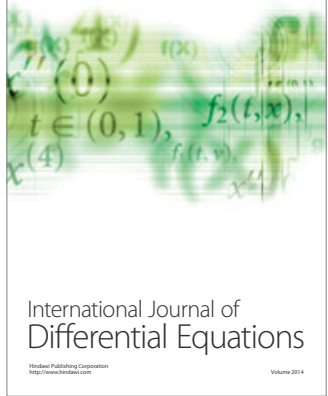
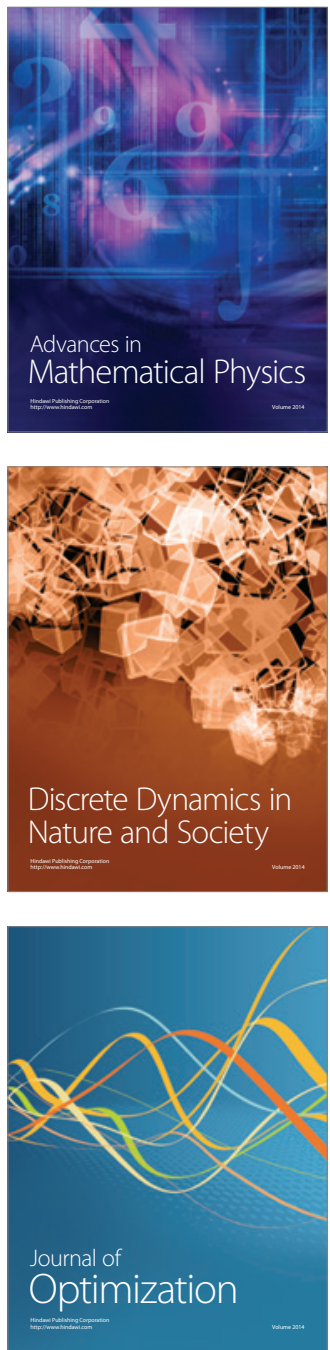